\title{
Hierarchical micro/nanostructured titanium with balanced actions to bacterial and mammalian cells for dental implants
}

This article was published in the following Dove Press journal:

International Journal of Nanomedicine

27 October 2015

Number of times this article has been viewed

\author{
Yu Zhul,* \\ Huiliang $\mathrm{Cao}^{2, *}$ \\ Shichong Qiaol,* \\ Manle Wang ${ }^{2,3}$ \\ Yingxin $\mathrm{Gu}^{\prime}$ \\ Huiwen Luo' \\ Fanhao Meng ${ }^{2}$ \\ Xuanyong Liu ${ }^{2}$ \\ Hongchang Lai' \\ 'Department of Oral and Maxillofacial \\ Implantology, Shanghai Key \\ Laboratory of Stomatology, Shanghai \\ Ninth People's Hospital, School \\ of Medicine, Shanghai Jiao Tong \\ University, ${ }^{2}$ State Key Laboratory \\ of High Performance Ceramics and \\ Superfine Microstructure, Shanghai \\ Institute of Ceramics, Chinese \\ Academy of Sciences, ${ }^{3}$ School of \\ Materials Engineering, Shanghai \\ University of Engineering Science, \\ Shanghai, People's Republic of China \\ *These authors contributed equally \\ to this work
}

Correspondence: Hongchang Lai Department of Oral and Maxillofacial Implantology, Shanghai Ninth People's Hospital, School of Medicine, Shanghai Jiao Tong University, 639 Zhizaoju Road, Shanghai 2000II, People's Republic of

China

Tel +86 2I 2327 I699 ext 5298

Fax +86 2I 53073068

Email lhc9@hotmail.com

Xuanyong Liu

Shanghai Institute of Ceramics, Chinese Academy of Sciences, No I 295 Ding-xi

Road, Shanghai 200050, People's Republic of China

Tel +862 2I 524I 2409

Fax +86 2I 524I 2409

Email xyliu@mail.sic.ac.cn
Abstract: A versatile strategy to endow dental implants with long-term antibacterial ability without compromising the cytocompatibility is highly desirable to combat implant-related infection. Silver nanoparticles (Ag NPs) have been utilized as a highly effective and broadspectrum antibacterial agent for surface modification of biomedical devices. However, the high mobility and subsequent hazardous effects of the particles on mammalian cells may limit its practical applications. Thus, Ag NPs were immobilized on the surface of sand-blasted, large grit, and acid-etched (SLA) titanium by manipulating the atomic-scale heating effect of silver plasma immersion ion implantation. The silver plasma immersion ion implantation-treated SLA surface gave rise to both good antibacterial activity and excellent compatibility with mammalian cells. The antibacterial activity rendered by the immobilized Ag NPs was assessed using Fusobacterium nucleatum and Staphylococcus aureus, commonly suspected pathogens for peri-implant disease. The immobilized Ag NPs offered a good defense against multiple cycles of bacteria attack in both $F$. nucleatum and $S$. aureus, and the mechanism was independent of silver release. F. nucleatum showed a higher susceptibility to $\mathrm{Ag}$ NPs than $S$. aureus, which might be explained by the presence of different wall structures. Moreover, the immobilized Ag NPs had no apparent toxic influence on the viability, proliferation, and differentiation of rat bone marrow mesenchymal stem cells. These results demonstrated that good bactericidal activity could be obtained with very small quantities of immobilized Ag NPs, which were not detrimental to the mammalian cells involved in the osseointegration process, and promising for titanium-based dental implants with commercial SLA surfaces.

Keywords: silver nanoparticles, surface modification, antibacterial effects, cytotoxicity, plasma immersion ion implantation

\section{Introduction}

Rough surfaces are commonly used for bone anchorage on titanium dental implants. ${ }^{1-3}$ Compared to the smooth one, roughed titanium demonstrates superior osseointegration properties. ${ }^{4,5}$ The commercially used sand-blasted, large grit, and acid-etched (SLA) procedure produces a rough surface with long-term stability, ${ }^{6-8}$ which is optimal to enhance bone-to-implant contact according to the consensus paper of Lang et al. ${ }^{9}$

Unfortunately, the increased surface roughness produced by SLA treatments could lead to greater bacterial adhesion. ${ }^{10,11}$ Once adhered, bacteria tend to aggregate in a hydrated polymeric matrix to form a biofilm on the implant, which is difficult for host defense and antimicrobial therapy to destroy. ${ }^{12}$ Such implant-related infections may lead to suppuration, revision surgery, and even removal of the device, all of which are associated with extremely high medical costs. ${ }^{13,14}$ It is well known that microorganisms and host cells compete for the substrate in a process called "race for the surface". 15,16 
Moreover, bacterial cells in the biofilms are more resistant to antimicrobial agents. ${ }^{17}$ Thus, an effective strategy to inhibit bacterial colonization is the local administration of an antibacterial agent on implant surfaces, which can inhibit adhesion of pioneer bacteria and kill them when they try to attach to the surface. ${ }^{18,19}$

Silver as a nonspecific biocidal agent is able to act strongly against a broad spectrum of bacterial and fungal species, including antibiotic-resistant strains. ${ }^{20}$ Silver nanoparticles (Ag NPs) are believed to be more reactive than the bulk metallic forms due to the larger active surface area. ${ }^{21}$ Therefore, various Ag NPs-modified films have been fabricated on dental implants for disinfection. ${ }^{22,23} \mathrm{Ag}$ NPs are known to interact with bacterial membrane constituents, cause structural changes, interrupt transmembrane electron transfer, oxidize bacterial components, and eventually induce bacterial death, ${ }^{24}$ whereas the exact mechanism is still controversial due to the difficulty in discerning the relative effects of all the possible antimicrobial actions rendered by Ag NPs.

Notably, the ultrasmall size and unique properties, such as high mobility of Ag NPs, also arouse concerns about their potential cytotoxicity. ${ }^{25}$ For instance, Zhao et $\mathrm{al}^{25}$ and De Giglio et $\mathrm{al}^{26}$ have reported that coatings incorporating Ag NPs exhibit some cytotoxicity toward osteoblasts, which is probably due to the silver ions $\left(\mathrm{Ag}^{+}\right)$released by Ag NPs. ${ }^{27}$ Hence, restricting the mobility of Ag NPs by immobilization is crucial in reducing the toxic effect. ${ }^{28}$ Silver plasma immersion ion implantation (Ag-PIII) is a nonline-of-light process, which is widely used in surface modifications to enhance antimicrobial efficacy. ${ }^{29}$ In our previous work, we found that the Ag-PIII technique with atomic-scale heating (ASH) effects could minimize the mobility of Ag NPs embedded in titanium, and these immobilized Ag NPs demonstrated outstanding compatibility to both soft and hard tissues. ${ }^{30}$

Accordingly, in this study, by manipulating the ASH effect of Ag-PIII, size tunable Ag NPs were immobilized on SLA-treated titanium to produce micro/nanostructural surfaces. The in vitro antimicrobial and compatible properties of these structures were investigated using the commonly suspected oral pathogens and rat bone marrow mesenchymal stem cells (rBMSCs), respectively.

\section{Material and methods Sample preparation}

Commercially pure titanium disks $(15 \mathrm{~mm}$ in diameter and $1 \mathrm{~mm}$ in thickness) of grade IV were cut and then sand blasted with large corundum grits (particle size 250-500 $\mu \mathrm{m}$ ) and acid etched in a boiling mixture of $\mathrm{HCl}$ and $\mathrm{H}_{2} \mathrm{SO}_{4}$ in order to obtain SLA surfaces (Trausim Medical Instrument Co., Ltd, Changzhou City, Jiangsu Province, People's Republic of China). Afterward, the disks were transferred into the chamber of anion implantation system, in which the SLA surface was further treated with Ag-PIII. A detailed description of the Ag-PIII process can be found in our previous study. ${ }^{30}$ The Ag-PIII parameters for the three sample groups involved in this study are listed in Table 1. Titanium disk treated only by SLA was used as a control.

\section{Surface chemistry and structure of the samples}

The surface roughness of all groups was assessed with a profilometer (Perthometer M1; Mahr, Göttingen, Germany). The surface morphologies of the samples before and after Ag-PIII were examined by field-emission scanning electron microscopy (SEM, JSM-6700F; JEOL, Tokyo, Japan). The chemical compositions of the surfaces were determined by X-ray photoelectron spectroscopy (XPS, PHI 5802; Physical Electronics Inc, Eden Prairie, MN, USA).

\section{Release of silver}

The Ag-PIII-treated samples were immersed in $10 \mathrm{~mL}$ of phosphate-buffered saline (PBS) at $37^{\circ} \mathrm{C}$ for up to 30 days without stirring. Then the PBS solutions containing the silver released from the Ag-PIII samples were analyzed by inductively coupled plasma mass spectrometry (Thermo X series 2).

Table I Surface modification details of the samples involved in the study

\begin{tabular}{lll}
\hline Groups & Samples & Surface modification details \\
\hline Test group I & 30 min-I5 Ag-PIII & SLA treated and Ag-PIII treated with a bias voltage of I5 kV for 30 minutes \\
Test group 2 & 60 min-I5 Ag-PIII & SLA treated and Ag-PIII treated with a bias voltage of I5 kV for 60 minutes \\
Test group 3 & 90 min-I5 Ag-PIII & SLA treated and Ag-PIII treated with a bias voltage of I5 kV for 90 minutes \\
Control group & SLA & SLA treated \\
\hline
\end{tabular}

Notes: 30 min-15 Ag-PIII, titanium surfaces treated by first SLA procedure and then silver plasma immersion ion implantation at 15 kV for 30 minutes; 60 min-15 Ag-PIII, titanium surfaces treated by first SLA procedure and then silver plasma immersion ion implantation at $15 \mathrm{kV}$ for 60 minutes; 90 min- $15 \mathrm{Ag}$-PIII, titanium surfaces treated by first SLA procedure and then silver plasma immersion ion implantation at $15 \mathrm{kV}$ for 90 minutes.

Abbreviations: Ag-PIII, silver plasma immersion ion implantation; SLA, sand-blasted, large grit, and acid etched; min, minutes. 


\section{Bacterial culture}

Fusobacterium nucleatum (ATCC 25586) and Staphylococcus aureus (ATCC 25923) were cultivated in the brain heart infusion broth medium at $37^{\circ} \mathrm{C}$. S. aureus was incubated on a rotary shaker $(250 \mathrm{rpm})$ under aerobic conditions, ${ }^{18}$ whereas $F$. nucleatum was incubated under standard anaerobic conditions $\left(80 \% \mathrm{~N}_{2}, 10 \% \mathrm{H}_{2}\right.$, and $\left.10 \% \mathrm{CO}_{2}\right) \cdot{ }^{31}$ Both of the bacteria were involved in the assays of various samples. The samples were placed on 24-well culture plates and separately incubated in $1 \mathrm{~mL}$ of the bacteria-containing medium $\left(10^{7}\right.$ colony-forming unit $[\mathrm{CFU}] / \mathrm{mL}$ for $F$. nucleatum and $10^{6} \mathrm{CFU} / \mathrm{mL}$ for $S$. aureus) for 24 hours. Before bacteria incubation, the specimens were sterilized in an autoclave at $121^{\circ} \mathrm{C}$ for 40 minutes. ${ }^{30}$

\section{Antibacterial assays by fluorescence staining}

After exposure to bacteria $\left(10^{7} \mathrm{CFU} / \mathrm{mL}\right.$ for $F$. nucleatum and $10^{6} \mathrm{CFU} / \mathrm{mL}$ for $S$. aureus) for 24 hours, the samples were rinsed twice with PBS, stained with $500 \mu \mathrm{L}$ of the combination dye of SYTO 9 and propidium iodide (PI, LIVE/DEAD BacLight bacteria viability kits; Thermo Fisher Scientific, Waltham, MA, USA) for 15 minutes in darkness, ${ }^{32}$ and then examined by confocal laser scanning microscopy (LSM 510 meta; Carl Zeiss Meditec AG, Jena, Germany).

\section{Antibacterial assays by the spread plate method}

After culturing for 24 hours in $1 \mathrm{~mL}$ of the bacteria-containing medium $\left(10^{7} \mathrm{CFU} / \mathrm{mL}\right.$ for $F$. nucleatum and $10^{6} \mathrm{CFU} / \mathrm{mL}$ for $S$. aureus), the unattached bacteria on various samples were removed by gently rinsing with PBS twice and the adherent bacteria were ultrasonically detached for 5 minutes in a $150 \mathrm{~W}$ ultrasonic bath (B3500S-MT; Branson Ultrasonics Co., Shanghai, People's Republic of China) at a frequency of $50 \mathrm{~Hz}$ after rapid vortex mixing (Vortex Genie 2; Scientific Industries, Bohemia, NY, USA) at the maximum power for 1 minute. ${ }^{33}$ The solutions were serially diluted and re-cultivated in triplicate on sheep blood agar plates (for F. nucleatum) or agar plates (for S. aureus) for 48 hours and 24 hours, respectively. ${ }^{24}$ The number of CFUs on the agar plates was counted in accordance with the National Standard of China GB/T 4789.2 protocol. The antibacterial rates of the adhered bacteria on the samples were determined by the following relationship: antibacterial rate $(\%)=(\mathrm{CFU}$ of control - CFU of experimental groups)/CFU of control $\times 100 \%$, where control indicated the average number of bacterial colonies associated with SLA surface and experimental groups indicated the average number of bacterial colonies associated with Ag-PIII samples. ${ }^{34}$

\section{Antibacterial assays by SEM observation}

After incubation in bacteria-containing medium $\left(10^{7} \mathrm{CFU} / \mathrm{mL}\right.$ for F. nucleatum and $10^{6} \mathrm{CFU} / \mathrm{mL}$ for $S$. aureus) for 24 hours, the samples were rinsed twice with PBS and fixed with 3\% glutaraldehyde at $4^{\circ} \mathrm{C}$ for 2 hours. Then all these samples were dehydrated sequentially in a series of ethanol solutions for 10 minutes each. ${ }^{35}$ Prior to SEM observation, the specimens were dried and sputter coated with platinum.

\section{Longevity and stability of the antibacterial activity}

To assess the long-term antibacterial activity, the samples were immersed in PBS for up to 30 days. At selected time points ( 1 day, 15 days, and 30 days), the samples were cultured in bacteria-containing medium $\left(10^{7} \mathrm{CFU} / \mathrm{mL}\right.$ for $F$. nucleatum and $10^{6} \mathrm{CFU} / \mathrm{mL}$ for S. aureus) for 24 hours, and the antibacterial rates of the Ag-PIII samples were calculated by the spread plate methods. To evaluate the stability of the antibacterial activity, the samples were incubated with the abovementioned bacterial suspension for 24 hours, ultrasonically cleaned, sterilized, and re-inoculated as aforementioned. This process was repeated for every 2 days for three times, and the antibacterial rates were calculated by the spread plate methods. ${ }^{24}$

\section{Cell culture}

rBMSCs were obtained from 4-week-old rats femurs, and in total ten rats were used to obtain sufficient cells for these experiments. The animal process protocols were approved by the animal research committee of the Ninth People's Hospital Affiliated with Shanghai Jiao Tong University School of Medicine. rBMSCs were isolated and cultured according to the protocol reported by Jiang et $\mathrm{al}^{36}$ and Zhang et al. ${ }^{37,38}$ Briefly, both ends of the femora were cut off at the epiphysis, and the marrow was flushed out using Dulbecco's Modified Eagle's Medium(DMEM); (Thermo Fisher Scientific, USA) with 10\% fetal bovine serum (Hyclone, USA). Then the isolated cells were incubated in DMEM supplemented with $10 \%$ fetal bovine serum in an incubator with an atmosphere of $5 \% \mathrm{CO}_{2}$ at $37^{\circ} \mathrm{C}$. After 72 hours, the nonadherent cells were rinsed away using PBS for several times. Then the culture medium was changed three times each week. When the confluence of $80 \%-90 \%$ was reached, the rBMSCs were subcultured into new dishes at a density of $1.0 \times 10^{5}$ cells $/ \mathrm{mL}$. The following experiments were carried out with two to three passages of cells. 


\section{Cell adhesion and spreading assay}

For the observation of the cytoskeletal actin, the $1.0 \mathrm{~mL}$ cell suspension with a cell density of $4 \times 10^{4} \mathrm{cell} / \mathrm{mL}$ was seeded onto all the samples. After incubation for 1 hour, 4 hours, and 24 hours, the rBMSCs on various samples were rinsed with PBS twice and fixed in $4 \%$ paraformaldehyde for 10 minutes at room temperature, followed by two rinses with PBS. Afterward, the cells on these samples were permeabilized with $0.5 \%$ Triton X-100 (Amresco, Solon, $\mathrm{OH}, \mathrm{USA}$ ) for 5 minutes at room temperature and rinsed with PBS twice. Then the cells were stained with $5 \mu \mathrm{g} / \mathrm{mL}$ fluorescein isothiocyanate-phalloidin (Sigma-Aldrich Co., St Louis, MO, USA) for 30 minutes under light protection and further stained for the cellular nuclei with 4',6-diamidino2-phenylindole dihydrochloride (DAPI; Sigma-Aldrich Co.) for 10 minutes. ${ }^{30,39,40}$ Subsequently, the F-actin and cell nuclei of rBMSCs on all the samples were observed by inverted fluorescence microscope (IX70; Olympus Corporation, Tokyo, Japan).

\section{Cytotoxicity assay}

The cytotoxicity of the samples on rat BMSCs was assayed by the LIVE/DEAD ${ }^{\circledR}$ viability/cytotoxicity kit for mammalian cells (Thermo Fisher Scientific). The kit contained two probes: calcein AM and ethidium homodimer-1. After the cells were seeded $\left(4.0 \times 10^{4}\right.$ cells $\left./ \mathrm{mL}\right)$ and incubated for 1 day, 3 days, and 5 days, the samples were taken from the 24-well plates and rinsed with PBS twice. Then $500 \mu \mathrm{L}$ of the work solution which is a mixture of $2 \mu \mathrm{M}$ calcein $\mathrm{AM}$ and $4 \mu \mathrm{M}$ ethidium homodimer-1, was immediately added to each sample and incubated in darkness at $37^{\circ} \mathrm{C}$ for 15 minutes. ${ }^{34}$ Subsequently, all the samples were rinsed with PBS and observed by inverted fluorescence microscope (IX70; Olympus Corporation).

\section{Cell proliferation assay}

The 3-(4,5-dimethyl-thiazol-2-yl)-2,5-diphenyl-tetrazolium bromide (MTT) assay (Sigma Chemical, Perth, Australia) was used to evaluate the proliferation activity of rBMSCs on different samples. Initially, $4.0 \times 10^{4}$ cells $/ \mathrm{mL}$ was seeded onto each sample in 24-well plates. After 1 day, 3 days, and 5 days of culture, MTT solution was added to each targeted well, and the specimens were incubated for 4 hours to form formazan. ${ }^{41}$ The formazan was dissolved in dimethylsulfoxide and measured at $490 \mathrm{~nm}$ using an ELX ultra microplate reader (BioTek, Winooski, VT, USA). The results were expressed as units of optical density (OD) absorbance values. The experiment was performed in triplicate.

\section{Alkaline phosphatase staining and activity assay}

The intracellular alkaline phosphatase (ALP) activity was evaluated qualitatively and quantitatively using commercially available kits. rBMSCs were seeded on the samples in 24-well plates at a density of $4 \times 10^{4}$ cells $/ \mathrm{mL}$. After 4 days and 7 days of culture, the cells on the samples were fixed with $4 \%$ paraformaldehyde, washed three times with PBS, and then stained using a BCIP/NBTALP Color Development Kit (Beyotime, Haimen, People's Republic of China). ${ }^{42}$ A semiquantitative analysis of ALP activity was performed according to the protocol reported by Gu et al. ${ }^{43,44}$ Briefly, it was assayed by measuring the transformation of $p$-nitrophenyl-phosphate (Sigma-Aldrich Co.) into $p$-nitrophenol (pNP). At 4 days and 7 days, the cells were lysed with $0.1 \%$ Triton X-100 for 4 hours of incubation at $37^{\circ} \mathrm{C}$. Then, a $100 \mu \mathrm{L}$ cell lysate sample was mixed with $100 \mu \mathrm{L}$ of freshly prepared $p$-nitrophenylphosphate substrate $(1 \mathrm{mg} / \mathrm{mL})$ and incubated in $37^{\circ} \mathrm{C}$ water bath for 30 minutes. Following this incubation, the amount of pNP produced was quantified by absorbance measurements at $405 \mathrm{~nm}$ (BioTek). Total protein was also calculated by assaying diluted samples of cell lysate using the Pierce BCA Protein Assay Kit (Thermo Fisher Scientific) according to the manufacturer's instructions. ALP activity was expressed as absorbance at $405 \mathrm{~nm}$ (OD value) per milligram of total cellular proteins. All experiments were done in triplicate.

\section{Statistical analysis}

The assays were performed in triplicate, and the data were expressed as means \pm standard deviations. Each experiment was repeated three times. A one-way analysis of variation combined with a Student-Newman-Keuls post hoc test was used to determine the level of significance. ${ }^{21} P<0.05$ was regarded to be significant, and $P<0.01$ was considered highly significant.

Table 2 Surface roughness of the sample groups involved in the study

\begin{tabular}{lll}
\hline Groups & Samples & Ra $(\mu \mathrm{m})$ \\
\hline Test group I & $30 \mathrm{~min}-15 \mathrm{Ag}$-PIII & $1.44 \pm 0.24$ \\
Test group 2 & $60 \mathrm{~min}-15 \mathrm{Ag}$-PIII & $1.45 \pm 0.14$ \\
Test group 3 & $90 \mathrm{~min}-15 \mathrm{Ag}$-PIII & $1.45 \pm 0.19$ \\
Control group & SLA & $1.45 \pm 0.20$ \\
\hline
\end{tabular}

Notes: No significant differences in Ra values was found between groups. All the data are expressed as means \pm standard deviations $(n=3) .30$ min- 15 Ag-PIII, titanium surfaces treated by first SLA procedure and then silver plasma immersion ion implantation at $15 \mathrm{kV}$ for 30 minutes; 60 min-15 Ag-PIII, titanium surfaces treated by first SLA procedure and then silver plasma immersion ion implantation at $15 \mathrm{kV}$ for 60 minutes; 90 min-15 Ag-PIII, titanium surfaces treated by first SLA procedure and then silver plasma immersion ion implantation at $15 \mathrm{kV}$ for 90 minutes.

Abbreviations: Ra, average surface roughness; Ag-PIII, silver plasma immersion ion implantation; SLA, sand-blasted, large grit, and acid etched; min, minutes. 


\section{Results}

\section{Material characterizations}

The average surface roughness $(\mathrm{Ra})$ for the sample groups is listed in Table 2. The Ra values of SLA group, titanium surfaces treated by first SLA procedure and then silver plasma immersion ion implantation at $15 \mathrm{kV}$ for 30 minutes (30 min-15 Ag-PIII), titanium surfaces treated by first SLA procedure and then silver plasma immersion ion implantation at $15 \mathrm{kV}$ for 60 minutes (60 min-15 Ag-PIII), and titanium surfaces treated by first SLA procedure and then silver plasma immersion ion implantation at $15 \mathrm{kV}$ for 90 minutes (90 min-15 Ag-PIII) were, respectively, $1.45 \pm 0.20 \mu \mathrm{m}$, $1.44 \pm 0.24 \mu \mathrm{m}, 1.45 \pm 0.14 \mu \mathrm{m}$, and $1.45 \pm 0.19 \mu \mathrm{m}$, which all belonged to the "moderately rough" category. ${ }^{6}$ No statistically significant differences $(P<0.05)$ were observed among the four groups in terms of Ra values.

The microstructural evolutions on the SLA-treated titanium before and after undergoing Ag-PIII at $15 \mathrm{kV}$ for 30 minutes, 60 minutes, and 90 minutes (designated as 30 min-15 Ag-PIII, 60 min-15 Ag-PIII, and 90 min-15 Ag-PIII, respectively) were examined by SEM (Figure 1). The original SLA surface with a moderately rough surface was covered with macropits $\sim 3-4 \mu \mathrm{m}$ in diameter, and each macropit was scattered with micropits $\sim 1 \mu \mathrm{m}$ in diameter (Figure 1A). Although rough on the microscale, the SLA surface appeared as a relatively smooth one on the nanoscale (the insert in Figure 1A). After Ag-PIII, the homogeneous microrough structures of SLA were not altered, whereas significantly different morphology in the nanoscale could be detected in all Ag-PIII samples. It is known that the precipitating habit of Ag NPs during Ag-PIII meets the classical nucleation theory, including a sequence of physical processes, such as supersaturation, nucleation, and growth, which implies that the existing form of Ag NPs in SLA surfaces can be regulated by optimizing the PIII parameters, such as bias voltage and duration..$^{45}$ As shown under higher magnification, silver is well imposed on all PIII samples in the form of NPs instead of film, thus forming a micron/nanohierarchical structure on titanium. Nevertheless, the size and the distribution of the particles were quite different among them. On 30 min-15 Ag-PIII, the particles were distributed with the similar morphology and a narrow size range of 4-6 nm (Figure 1B). As the Ag-PIII duration increased, the density became higher but the average size of the particle remains almost unchanged (Figure 1C). When the Ag-PIII time increased to 90 minutes, the density of the particle increased, but the distribution of the particle diameter became bimodal with one peak at merely $2 \mathrm{~nm}$ and the other at 11-12 nm (Figure 1D).

To further determine the changes in chemical states of the sample surfaces, the control SLA group and the Ag-PIII-treated samples were analyzed by XPS. As the XPS full spectra is
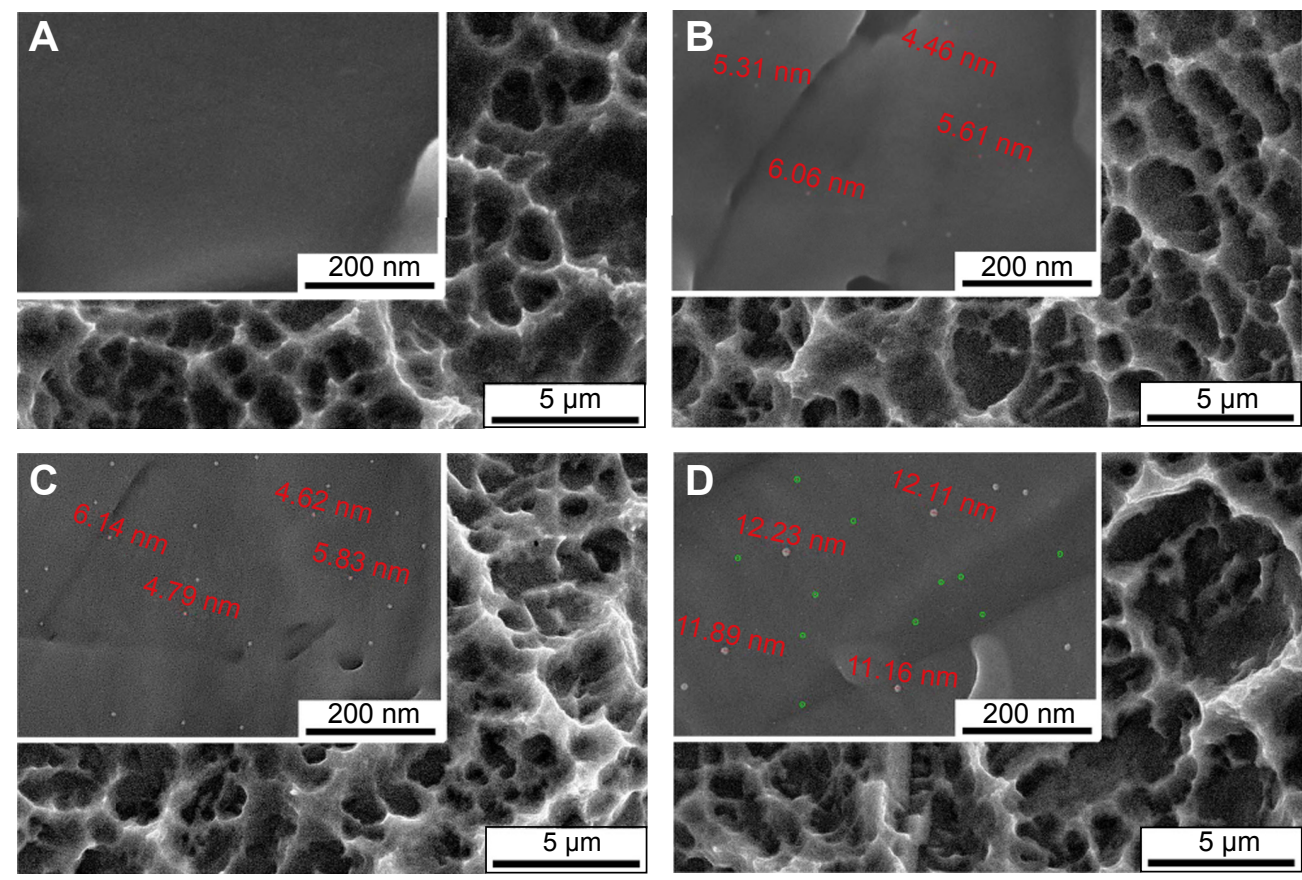

Figure I Surface microstructures of (A) SLA, (B) 30 min-15 Ag-PIII, (C) 60 min-I5 Ag-PIII, and (D) 90 min-15 Ag-PIII detected by SEM at low and high magnifications, respectively.

Notes: 30 min-15 Ag-PIII, titanium surfaces treated by first SLA procedure and then silver plasma immersion ion implantation at I5 kV for 30 minutes; 60 min-I5 Ag-PIII, titanium surfaces treated by first SLA procedure and then silver plasma immersion ion implantation at $15 \mathrm{kV}$ for 60 minutes; 90 min-I5 Ag-PIII, titanium surfaces treated by first SLA procedure and then silver plasma immersion ion implantation at $15 \mathrm{kV}$ for 90 minutes.

Abbreviations: SLA, sand-blasted, large grit, and acid etched; Ag-PIII, silver plasma immersion ion implantation; SEM, scanning electron microscopy; min, minutes. 
displayed in Figure 2A, titanium (Ti), oxygen (O), and carbon (C) were detected in the SLA control, indicating that a SLAfabricated titanium oxide layer was formed on titanium after SLA. The existence of silver (Ag) was confirmed on all the Ag-PIII groups, but no obvious differences were detected in the surface chemistry of these Ag-PIII samples. The amounts of Ag increased as the Ag-PIII duration prolonged, but they were remained at relatively low levels with only 0.31 at.\% for $30 \mathrm{~min}-15 \mathrm{Ag}$-PIII (Figure 2B), 0.39 at.\% for $60 \mathrm{~min}-$ 15 Ag-PIII (Figure 2C), and 0.45 at. $\%$ for $90 \mathrm{~min}-15 \mathrm{Ag}$-PIII (Figure 2D). In addition, as evidenced by the high-resolution XPS spectra (Figure 3), the Ag 3d doublet at $374.3 \mathrm{eV}$ (Ag 3d3/2) and $368.3 \mathrm{eV}$ (Ag 3d5/2) with a spin energy separation of $6.0 \mathrm{eV}$ correspond to metallic silver $\left(\mathrm{Ag}^{0}\right),{ }^{46}$ and there was no evidence of silver oxides, indicating that the NPs observed by SEM (Figure 1B-D) were in a metallic state.

\section{Silver release}

Instability of Ag-based bactericides can limit their applications, and the main influencing factor is that Ag can be
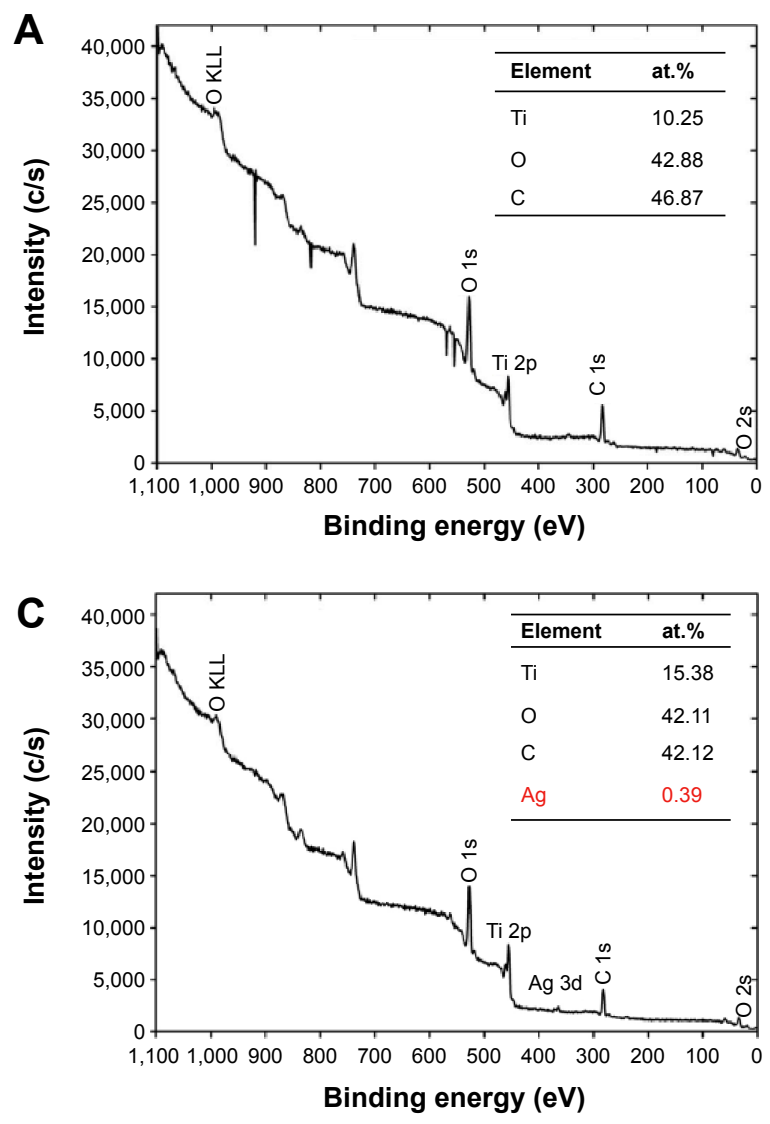

readily oxidized to silver ion $\left(\mathrm{Ag}^{+}\right)$, leading to inefficiency after long-term storage and causing environmental hazard. ${ }^{47}$ Accordingly, silver released from the Ag-PIII samples to PBS after a 30-day incubation was evaluated by inductively coupled plasma mass spectrometry. As shown in Figure 4, the amounts of silver released from 30 min-15 Ag-PIII, $60 \mathrm{~min}-15 \mathrm{Ag}$-PIII, and $90 \mathrm{~min}-15 \mathrm{Ag}$-PIII were $3.6 \mathrm{ppb}$, $4.3 \mathrm{ppb}$, and $4.6 \mathrm{ppb}$, respectively, signifying that the leakages of silver from Ag-PIII samples were minimal.

\section{Antimicrobial performance}

$S$. aureus (gram positive) and F. nucleatum (gram negative) have been identified as two clinically relevant bacteria in the implant-related infection. ${ }^{48}$ Thus, we evaluated the antimicrobial activities of the Ag-PIII groups against $S$. aureus and $F$. nucleatum at the first 24 hour using Live/Dead staining assays, spread plate methods, and SEM observation.

The Live/Dead assay is an effective method to visualize the living and dead bacteria based on the color change during the experiment. ${ }^{49}$ Figure 5 depicts the distribution
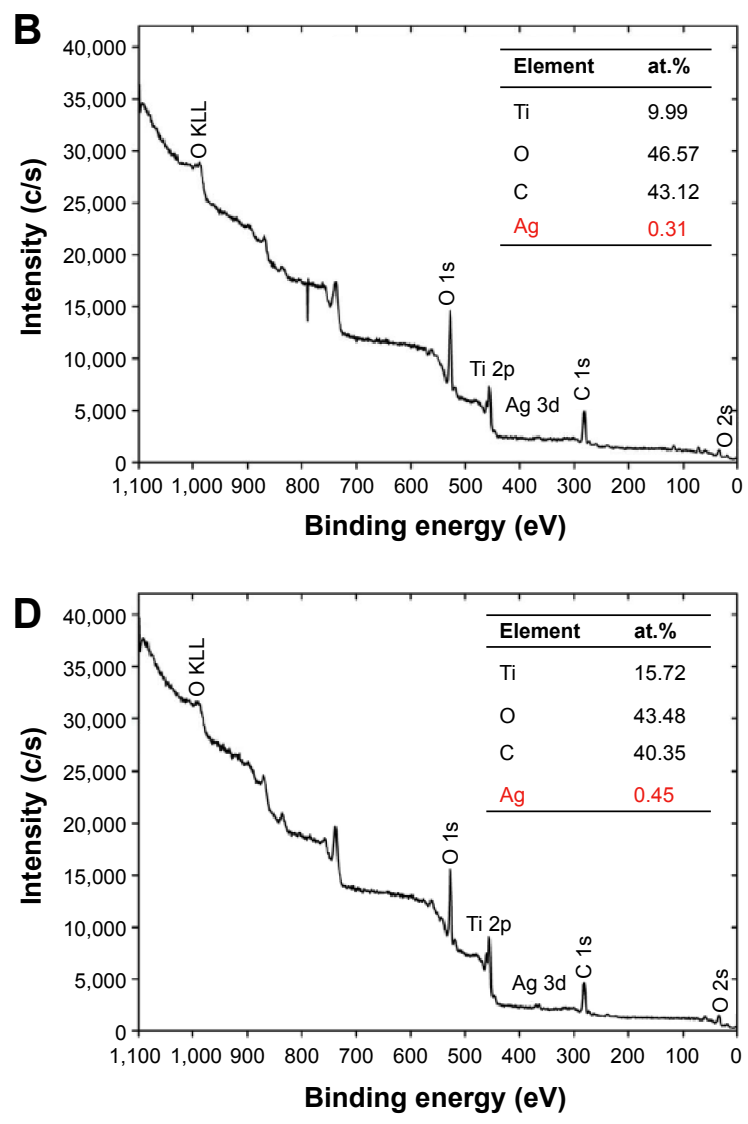

Figure 2 XPS full spectra of (A) SLA, (B) 30 min-15 Ag-PIII, (C) 60 min-15 Ag-PIII, and (D) 90 min-15 Ag-PIII.

Notes: 30 min-15 Ag-PIII, titanium surfaces treated by first SLA procedure and then silver plasma immersion ion implantation at $15 \mathrm{kV}$ for 30 minutes; 60 min- 15 Ag-PIII, titanium surfaces treated by first SLA procedure and then silver plasma immersion ion implantation at $15 \mathrm{kV}$ for 60 minutes; 90 min- $15 \mathrm{Ag}$-PIII, titanium surfaces treated by first SLA procedure and then silver plasma immersion ion implantation at $15 \mathrm{kV}$ for 90 minutes.

Abbreviations: XPS, X-ray photoelectron spectroscopy; SLA, sand-blasted, large grit, and acid etched; Ag-PIII, silver plasma immersion ion implantation; min, minutes. 

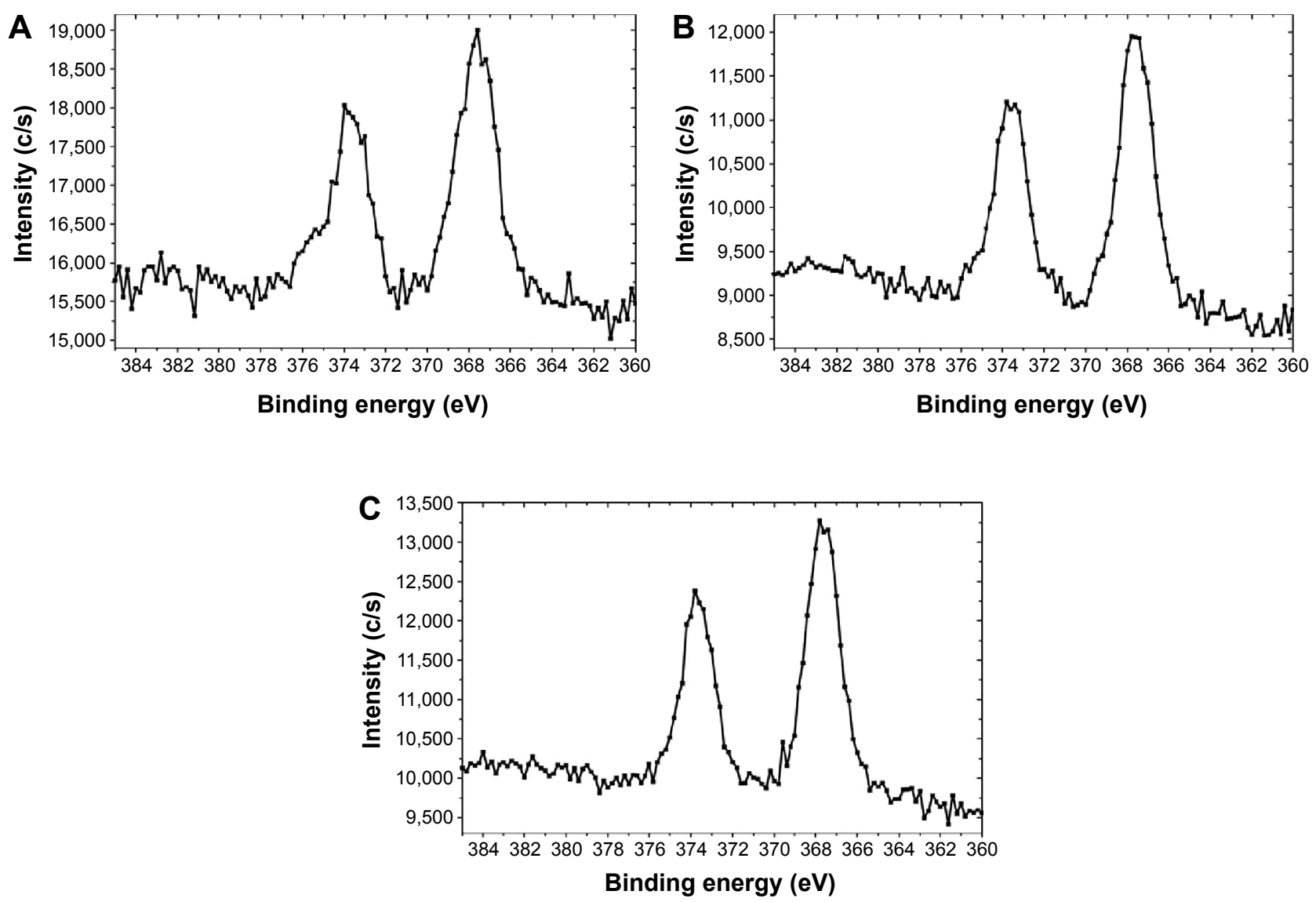

Figure 3 Ag 3d XPS spectra of the Ag-PIII groups: (A) 30 min-15 Ag-PIII, (B) 60 min-I5 Ag-PIII, and (C) 90 min-I5 Ag-PIII.

Notes: The peaks can be indexed to the typical binding energies for $\mathrm{Ag} 3 \mathrm{~d} 3 / 2$ and $\mathrm{Ag} 3 \mathrm{~d} 5 / 2$ in $\mathrm{TiO}_{2}$, respectively. 30 min- $15 \mathrm{Ag}-\mathrm{PIII}$, titanium surfaces treated by first SLA procedure and then silver plasma immersion ion implantation at $15 \mathrm{kV}$ for 30 minutes; 60 min- 15 Ag-PIII, titanium surfaces treated by first SLA procedure and then silver plasma immersion ion implantation at $15 \mathrm{kV}$ for 60 minutes; 90 min-15 Ag-PIII, titanium surfaces treated by first SLA procedure and then silver plasma immersion ion implantation at $15 \mathrm{kV}$ for 90 minutes.

Abbreviations: XPS, X-ray photoelectron spectroscopy; Ag-PIII, silver plasma immersion ion implantation; $\mathrm{Ag} 3 \mathrm{~d} 3 / 2, \mathrm{Ag} 3 \mathrm{~d}$ doublet at 374.3 eV; $\mathrm{Ag} 3 \mathrm{~d} 5 / 2$, Ag 3d doublet at $368.3 \mathrm{eV} ; \mathrm{TiO}_{2}$, titanium dioxide; SLA, sand-blasted, large grit, and acid etched; min, minutes.

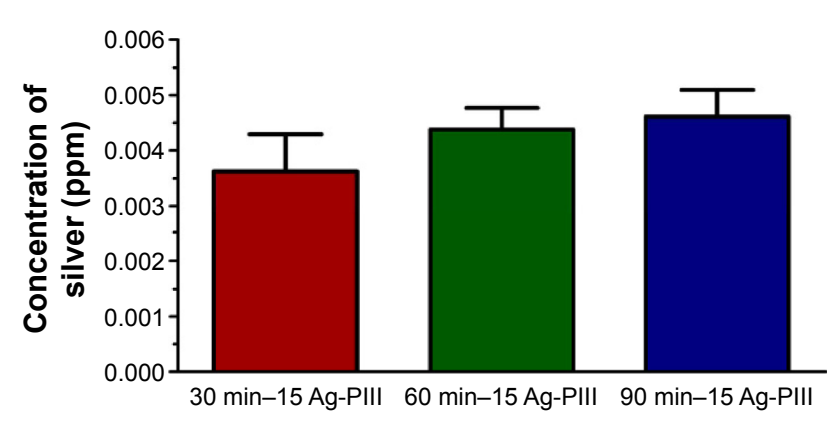

Figure 4 The silver release after soaking the Ag-PIII groups in PBS for 30 days was examined by ICP-MS.

Notes: Less than 5 ppb silver can be detected in Ag-PIII groups after soaking in PBS for 30 days. No significant differences in silver release are found between Ag-PIII groups. All the data are expressed as means \pm standard deviations $(n=3) .30$ min-15 Ag-PIII, titanium surfaces treated by first SLA procedure and then silver plasma immersion ion implantation at $15 \mathrm{kV}$ for 30 minutes; 60 min-15 Ag-PIII, titanium surfaces treated by first SLA procedure and then silver plasma immersion ion implantation at $15 \mathrm{kV}$ for 60 minutes; 90 min- $15 \mathrm{Ag}$-PIII, titanium surfaces treated by first SLA procedure and then silver plasma immersion ion implantation at $15 \mathrm{kV}$ for 90 minutes.

Abbreviations: Ag-PIII, silver plasma immersion ion implantation; ICP-MS, inductively coupled plasma mass spectrometry; PBS, phosphate-buffered saline; SLA, sand-blasted, large grit, and acid etched; min, minutes. of viable and dead bacteria, which adhered on the surfaces of the samples after immersion in the bacterial suspension for 1 day. SYTO 9 stained all bacteria green (those with intact membranes and those with damaged membranes), while PI penetrates only bacteria with damaged membranes, staining them red. ${ }^{39}$ As for $S$. aureus, the red spots on SLA surface were negligible, and there were many green spots, indicating the inability of SLA control in killing bacteria. Conversely, more red spots were observed from $60 \mathrm{~min}-$ 15 Ag-PIII, and there were less green spots, indicating improved antibacterial ability of $60 \mathrm{~min}-15 \mathrm{Ag}$-PIII against S. aureus. Significantly, large quantities of red spots and a relatively small number of green spots were presented on 90 min-15 Ag-PIII, revealing the best antibacterial effects among the Ag-PIII groups. Although the alteration in 30 min-15 Ag-PIII was not obvious, the total number of live bacteria colonies did decrease when compared with the SLA control. Similar results were obtained from $F$. nucleatum, 

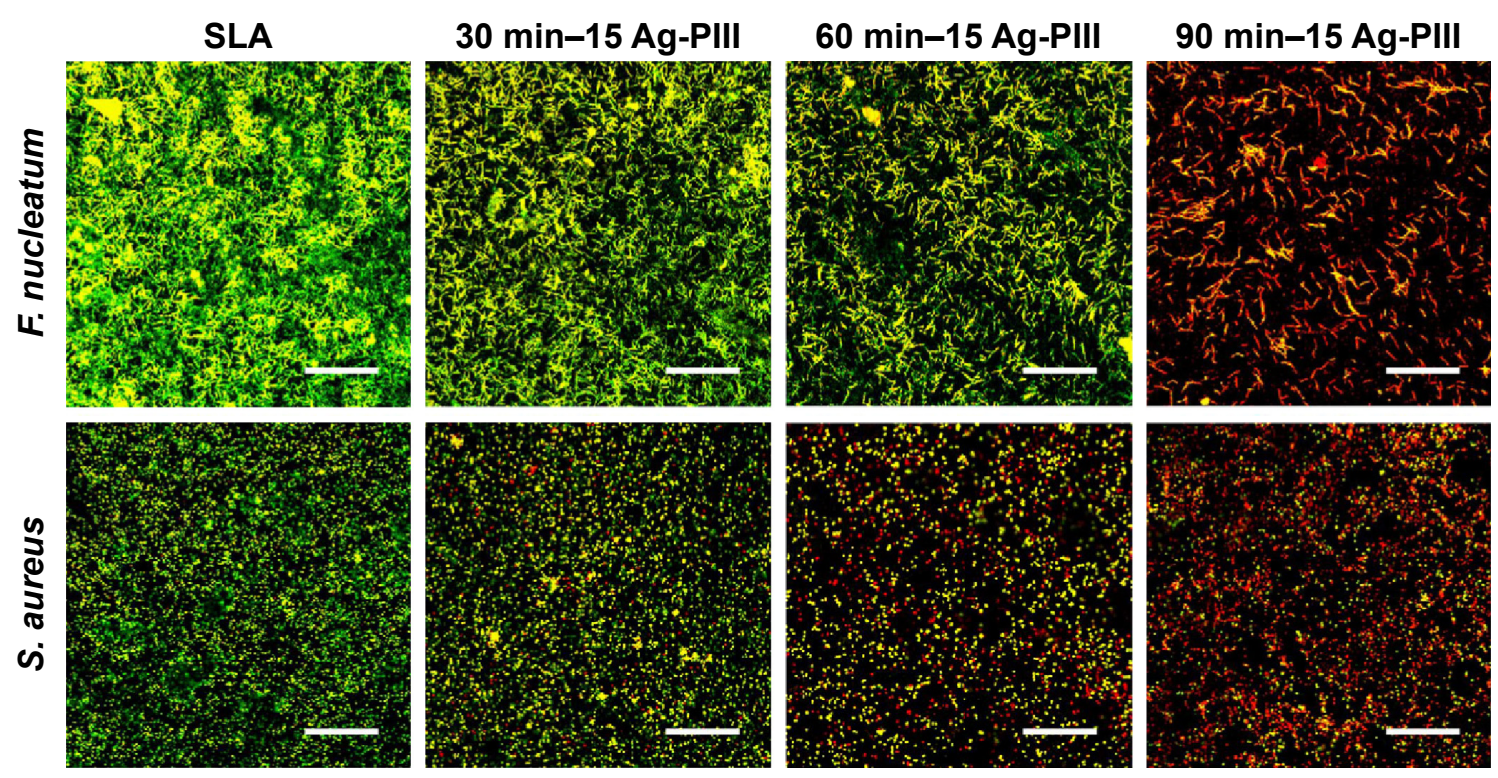

Figure 5 Fluorescent images of F. nucleatum and S. aureus cultured on SLA, 30 min-15 Ag-PIII, 60 min-I5 Ag-PIII, and 90 min-I5 Ag-PIII for 24 h, obtained by CLSM after staining with SYTO 9 and propidium iodide.

Notes: The scale bar represents $75 \mu \mathrm{m} .30 \mathrm{~min}-15 \mathrm{Ag}$-PIII, titanium surfaces treated by first SLA procedure and then silver plasma immersion ion implantation at $15 \mathrm{kV}$ for 30 minutes; 60 min-15 Ag-PIII, titanium surfaces treated by first SLA procedure and then silver plasma immersion ion implantation at I5 kV for 60 minutes; 90 min- 15 Ag-PIII, titanium surfaces treated by first SLA procedure and then silver plasma immersion ion implantation at $15 \mathrm{kV}$ for 90 minutes.

Abbreviations: SLA, sand-blasted, large grit, and acid etched; Ag-PIII, silver plasma immersion ion implantation; h, hours; CLSM, confocal laser scanning microscopy; min, minutes.

demonstrating good antibacterial activity of Ag-PIII groups, and particularly 90 min-15 Ag-PIII samples that possessed Ag NPs with relatively high density and big size, in killing both bacteria on contact (as aforementioned the leakages of silver from Ag-PIII groups were minimal).

It is noteworthy some of the live bacteria stained green were likely to be those that had adhered to the spots without the NPs or onto other bacteria that were in contact with the surface. Moreover, under certain conditions, bacteria with undamaged membranes were unable to duplicate, and then the number of live bacteria obtained by subtracting "red cells" from "green cells" accounted for both nonviable live (which may not duplicate) and viable (which can duplicate) bacteria. ${ }^{39}$ Although the cell wall of some bacteria remains intact after being exposed to the Ag NPs-incorporated surfaces, the number of bacteria capable of replicating and hence able to form a biofilm was only a fraction of the live bacterial population. ${ }^{18,50}$ In contrast to the Live/Dead assay, the spread plate methods reveal only viable bacteria, which was more precise and reliable.

To further investigate the antibacterial ability, the adhered bacteria were detached from the samples and re-cultured on agar plates in accordance with the bacteria counting methods, and the results are presented in Figure 6. The amounts of viable $F$. nucleatum and $S$. aureus on the SLA surface were very large, but those on Ag-PIII groups were much fewer, demonstrating that these Ag NPs were prone to reduce the proliferation of both pathogenic microbes. The antibacterial ability of the concerned groups ranked as follows: $90 \mathrm{~min}-15 \mathrm{Ag}$-PIII $>60 \mathrm{~min}-15 \mathrm{Ag}$-PIII $>30$ min-15 Ag-PIII > SLA, which indicated that the antimicrobial efficacy of Ag-PIII groups was probably dependent on the size and density of Ag NPs. Notably, the antibacterial activity of Ag NPs against $F$. nucleatum was superior to $S$. aureus, for the reduction rates of $F$. nucleatum on the three Ag-PIII groups were $\sim 22 \%, 57 \%$, and $92 \%$, respectively, whereas those of $S$. aureus were $12 \%, 42 \%$, and $80 \%$, respectively.

SEM observation was performed to examine the morphology and membrane integrity of the both pathogenic bacteria. As shown in Figure 7, a large amount of $F$. nucleatum cells was found on the SLA group, while the number of bacteria was obviously decreased on Ag-PIII groups. The bacterial cells on pure SLA had mostly the rod shape, whereas those on 30 min-15 Ag-PIII were corrugated with a distorted shape, and the membrane contrast was not as sharp as that on the SLA control. Apparent cell debris were observed from 60 min-15 Ag-PIII, and some of the cells even merge with the background, showing that $F$. nucleatum could hardly survive on 60 min-15 Ag-PIII. The bacteria on 90 min-15 Ag-PIII were completely lysed, and cells with a normal shape could be hardly found, indicating that $90 \mathrm{~min}-15 \mathrm{Ag}$-PIII had the best bacterial activity against $F$. nucleatum. Similarly, S. aureus cells proliferated well and formed abundant biofilms on SLA, 


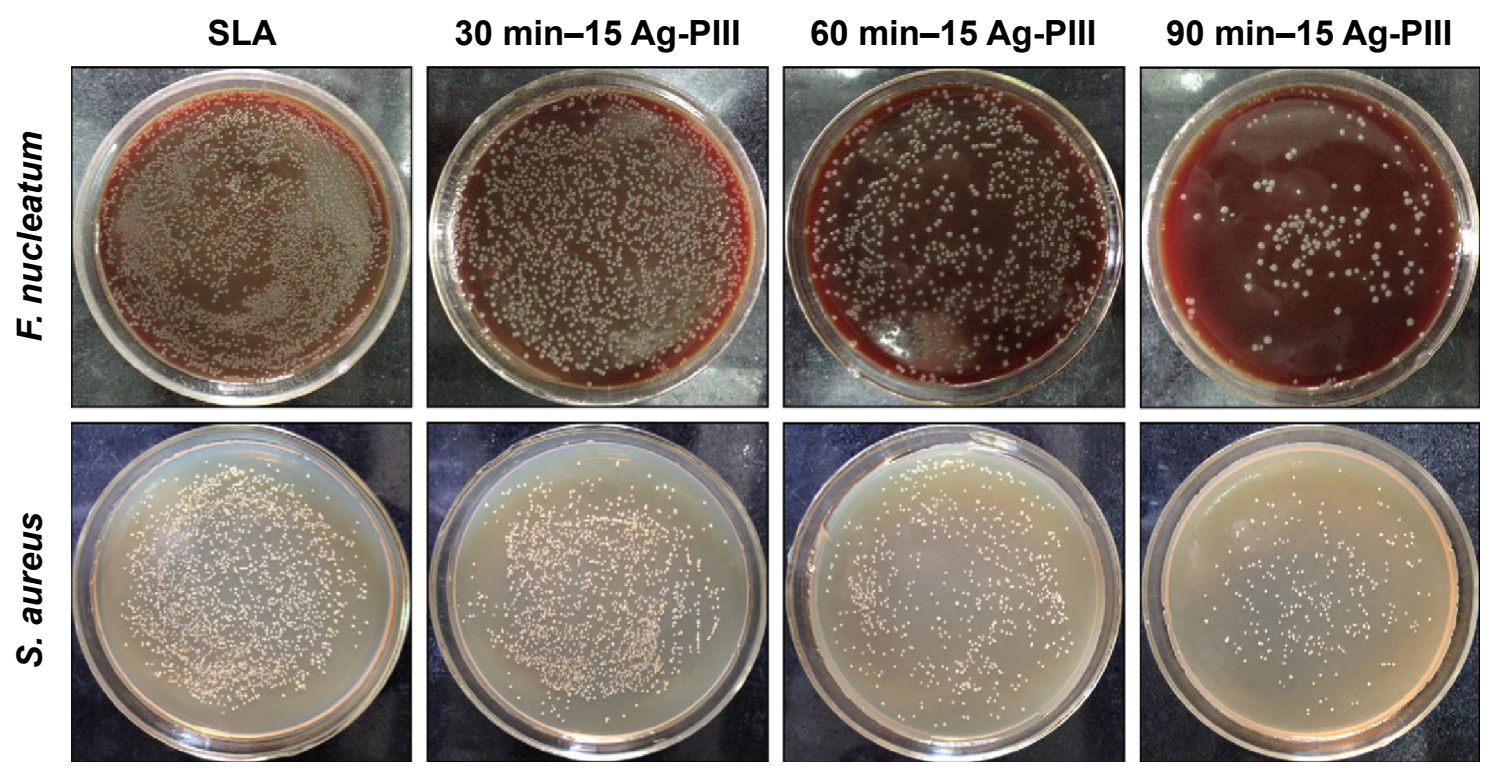

Figure 6 Re-cultivated microbial colonies on agar plates: F. nucleatum and S. aureus were detached from SLA, 30 min-I5 Ag-PIII, 60 min-I5 Ag-PIII, and 90 min-I5 Ag-PIII, respectively.

Notes: 30 min-15 Ag-PIII, titanium surfaces treated by first SLA procedure and then silver plasma immersion ion implantation at I5 kV for 30 minutes; 60 min- I5 Ag-PIII, titanium surfaces treated by first SLA procedure and then silver plasma immersion ion implantation at $15 \mathrm{kV}$ for 60 minutes; 90 min-15 Ag-PIII, titanium surfaces treated by first SLA procedure and then silver plasma immersion ion implantation at $15 \mathrm{kV}$ for 90 minutes.

Abbreviations: SLA, sand-blasted, large grit, and acid etched; Ag-PIIl, silver plasma immersion ion implantation; min, minutes.

whereas the number of bacteria was significantly reduced on Ag-PIII. The S. aureus cells had a spherical shape with a smooth surface on SLA, whereas those on 30 min-15 Ag-PIII were highly dispersed with rougher surfaces. Abnormal cell shape distortion and prevalent cell lysis could be observed from 60 min-15 Ag-PIII, whereas completely lysed bacteria were observed from 90 min-15 Ag-PIII. These results implied that the death of both F. nucleatum and S. aureus was partly due to the disruption of the membrane integrity and lysis of bacteria.
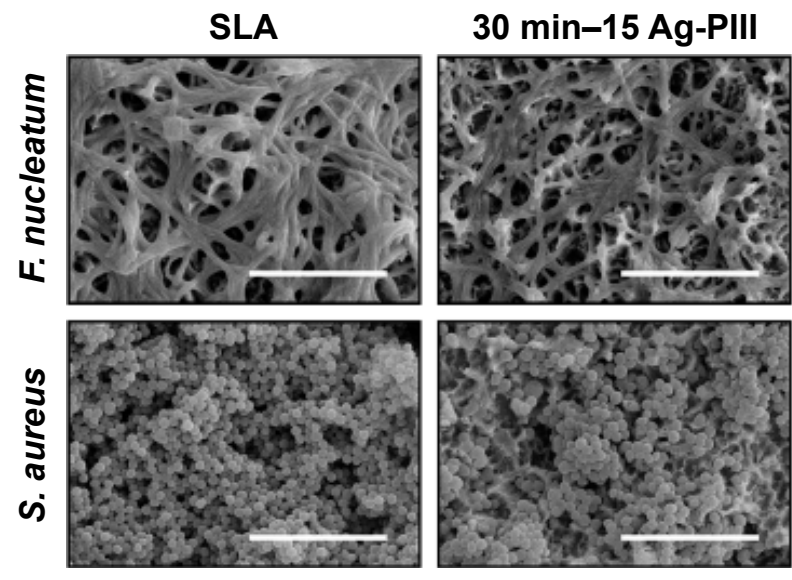

\section{Longevity and stability of the antibiofilm property}

Longevity and stability of an antibacterial strategy were crucial to clinical adoption, because bacterial contaminations may occur not only perioperatively but also hematogenously later during the lifetime of the implant. ${ }^{51}$ Thus, the longterm antibacterial activity of Ag-PIII groups was assessed. As shown in Figure 8A, the antibacterial rates of $30 \mathrm{~min}-$ 15 Ag-PIII, 60 min-15 Ag-PIII, and 90 min-15 Ag-PIII against $S$. aureus and F. nucleatum remain at $82.1 \%-85.9 \%$,

Figure 7 SEM morphologies of Fusobacterium nucleatum and Staphylococcus aureus seeded on SLA, 30 min-15 Ag-PIII, 60 min-I5 Ag-PIII, and 90 min-I5 Ag-PIII.

Notes: The scale bar represents $10 \mu \mathrm{m}$. $30 \mathrm{~min}-15 \mathrm{Ag}$-PIII, titanium surfaces treated by first SLA procedure and then silver plasma immersion ion implantation at I5 kV for 30 minutes; 60 min-I5 Ag-PIII, titanium surfaces treated by first SLA procedure and then silver plasma immersion ion implantation at I5 kV for 60 minutes; 90 min- 15 Ag-PIII, titanium surfaces treated by first SLA procedure and then silver plasma immersion ion implantation at $15 \mathrm{kV}$ for 90 minutes.

Abbreviations: SEM, scanning electron microscopy; SLA, sand-blasted, large grit, and acid etched; Ag-PIII, silver plasma immersion ion implantation; min, minutes. 


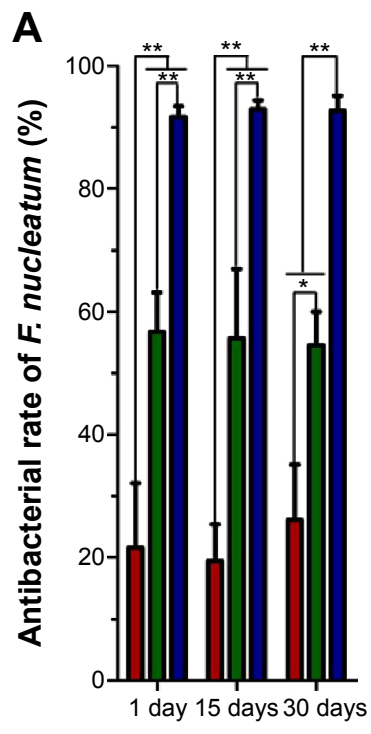

Culture time

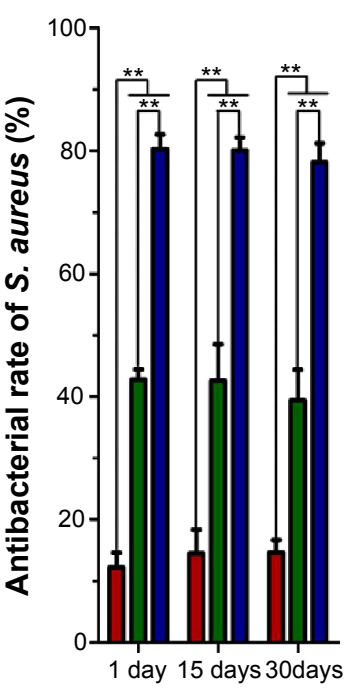

Culture time
B

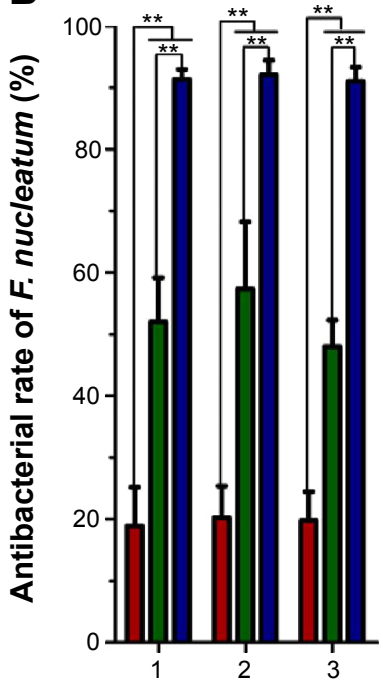

Times of antibacterial

trial

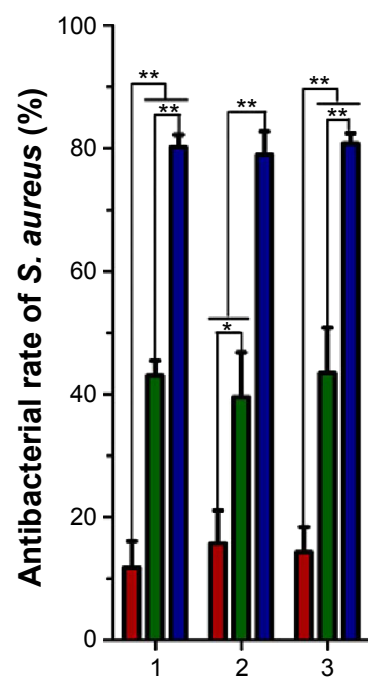

Times of antibacterial trial

30 min-15 Ag-PIII 60 min-15 Ag-PIII

90 min-15 Ag-PIII

Figure 8 Antibacterial rates of the Ag-PIII groups after being incubated in PBS for I day, I5 days, and 30 days (A) and repeated bacterial attack (B).

Notes: No significant changes are found from the antibacterial rates of the Ag-PIII groups after incubation in PBS for up to 30 days and three cycles of bacteria exposure. All the data are expressed as means \pm standard deviations $(n=3)$. $* P<0.05$ and $* * P<0.01 .30$ min-15 Ag-PIII, titanium surfaces treated by first SLA procedure and then silver plasma immersion ion implantation at $15 \mathrm{kV}$ for 30 minutes; 60 min- $15 \mathrm{Ag}$-PIII, titanium surfaces treated by first SLA procedure and then silver plasma immersion ion implantation at $15 \mathrm{kV}$ for 60 minutes; 90 min-15 Ag-PIII, titanium surfaces treated by first SLA procedure and then silver plasma immersion ion implantation at $15 \mathrm{kV}$ for 90 minutes. Abbreviations: Ag-PIII, silver plasma immersion ion implantation; PBS, phosphate-buffered saline; SLA, sand-blasted, large grit, and acid etched; min, minutes.

$85.4 \%-91.7 \%$, and $92.6 \%-94.4 \%$ and $82.1 \%-85.9 \%$, $85.4 \%-91.7 \%$, and $92.6 \%-94.4 \%$, respectively. No significant differences $(P>0.05)$ in antibacterial rates are found in the same Ag-PIII group after incubation in PBS for up to 30 days, which indicated that the antibacterial activity of Ag-PIII groups could be sustained and long lasting. Moreover, the capability of the Ag-PIII in multiple bacteria attacks was also evaluated. As shown in Figure 8B, the antibacterial rates of the Ag-PIII samples do not change significantly $(P>0.05)$ after three cycles of bacteria exposure, suggesting that the Ag-PIII groups had stable antibacterial activity.

\section{Cytocompatibility}

Taking into account the clinical implications of using Ag NPs as antimicrobial agents, the question that immediately arises is whether the dosages of NPs are in the concentration range at which they are effective to kill bacteria but do no harm to mammalian cells. ${ }^{52}$ In fact, it was reported that Ag NPs could reduce the mitochondrial functions and also affect the membrane integrity of mammalian cells. ${ }^{18}$ In addition, Ag NPs were identified as responsible for the production of reactive oxygen species and interruption of adenosine triphosphate synthesis, which, in turn, cause DNA damage. ${ }^{18}$ Thus, given its potential cytotoxicity, we have tested the cytocompatibility of Ag-PIII groups with
rBMSCs, the most well-characterized cell source involved in osseointegration.

The initial cell adhesion and spreading activity were assayed by staining with fluorescein isothiocyanate and DAPI to visualize the F-actin and nuclei, respectively. As shown in Figure 9, the rBMSCs on all groups exhibited a spherical morphology in the first hour due to the lack of filopodia extensions and mitosis phase cells. After culturing for 4 hours, the expressions of F-actin on all groups were better than those in the first hour, and a lot of filopodia could be detected. After culturing for 24 hours, the difference in the expressions of F-actin was still not significant, and the rBMSCs on all surfaces exhibited multipolar spindle morphologies with well-organized cytoskeleton structures as well as numerous filopodia and lamellipodia. These results indicated that the rBMSCs acclimated rapidly to all the samples, and Ag NPs produced by Ag-PIII did no harm to the initial adhesion and spreading activity of rBMSCs. This was likely due to the restricted mobility of the particles that exclude the uptake of rBMSCs through endocytosis and macropinocytosis, for uptake of Ag NPs may inhibit normal cell function. ${ }^{53}$

Based on the common concern that cellular uptake of Ag NPs may inhibit cell growth, ${ }^{53}$ we assessed the vitality and proliferation of rBMSCs on all the samples qualitatively using the Live/Dead assay and quantitatively using 


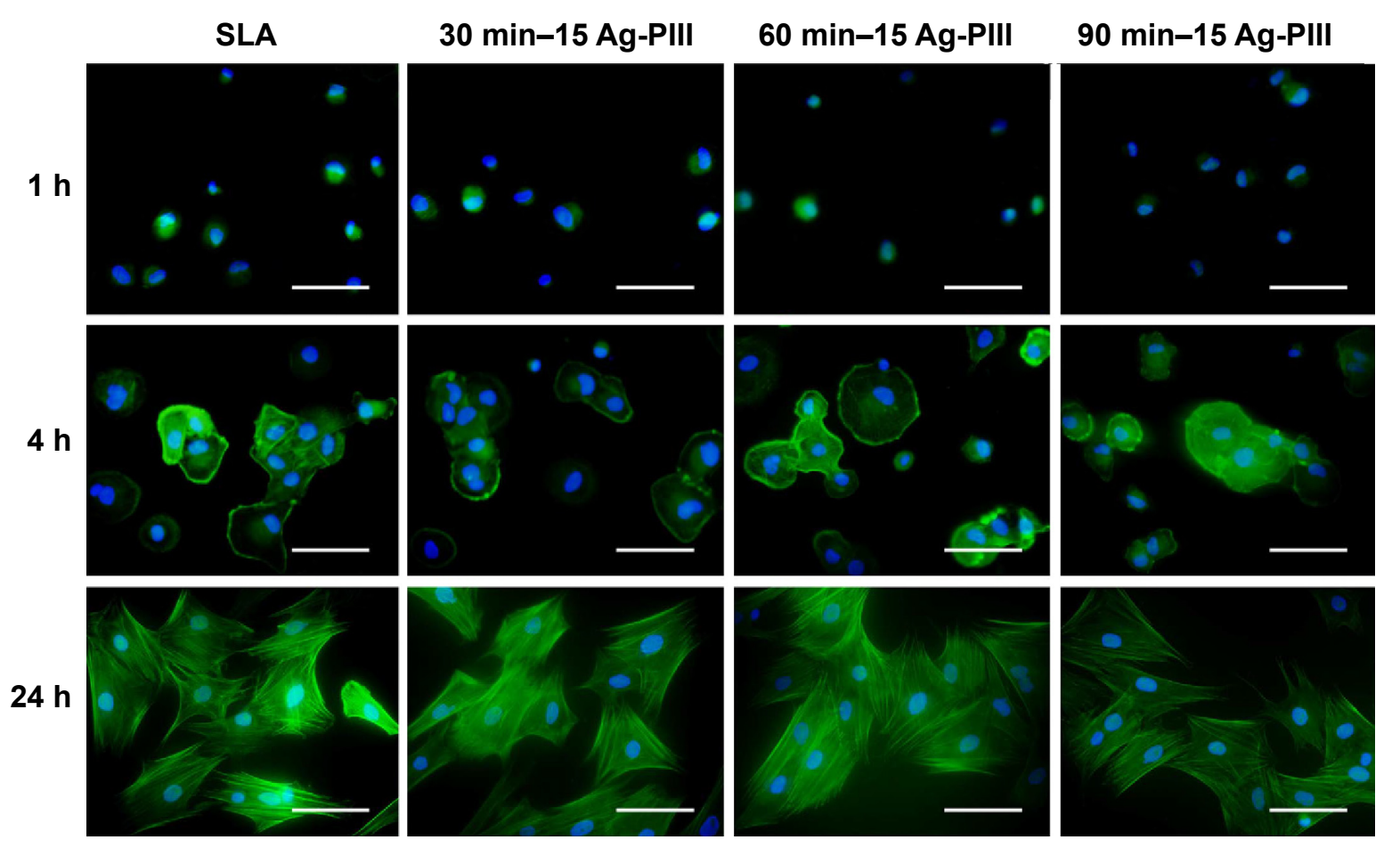

Figure 9 Fluorescent images of rBMSCs cultured on SLA, 30 min-15 Ag-PIII, 60 min-I5 Ag-PIII, and 90 min-I5 Ag-PIII for I h, 4 h, and 24 h with F-actin stained with FITC (green) and nuclei stained with DAPI (blue).

Notes: The scale bar represents $100 \mu \mathrm{m}$. 30 min-15 Ag-PIII, titanium surfaces treated by first SLA procedure and then silver plasma immersion ion implantation at $15 \mathrm{kV}$ for 30 minutes; 60 min-15 Ag-PIII, titanium surfaces treated by first SLA procedure and then silver plasma immersion ion implantation at I5 kV for 60 minutes; 90 min-15 Ag-PIII, titanium surfaces treated by first SLA procedure and then silver plasma immersion ion implantation at $15 \mathrm{kV}$ for 90 minutes.

Abbreviations: rBMSCs, rat bone marrow mesenchymal stem cells; SLA, sand-blasted, large grit, and acid etched; Ag-PIII, silver plasma immersion ion implantation; FITC, fluorescein isothiocyanate; DAPI, 4',6-diamidino-2-phenylindole dihydrochloride; h, hours; min, minutes.

the MTT assay after culturing for 1 day, 3 days, and 5 days. In the Live/Dead assay, the Calcein AM could be converted to calcein by active intracellular esterase of living cells to generate green fluorescence, whereas PI could only enter dead cells with broken cell membrane and produce red fluorescence after binding to the nucleic acids. As shown in Figure 10A, almost no dead cells can be observed in all the sample groups, and the number of cells increases linearly as the culture duration prolonged. The results of MTT assay were remarkably consistent with the Live/Dead staining results. As shown in Figure 10B, the average OD values increase with culture time on account of continuous cell proliferation. No significant difference $(P>0.05)$ in cell proliferation was observed among all the sample groups. The results consistently indicated that the Ag-PIII groups had no apparent cytotoxicity and could support the viability and proliferation of rBMSCs.

In view that the cellular uptake of Ag NPs may impose adverse effects on osteoblastic differentiation, ${ }^{53}$ the activity of ALP, a typical differentiation marker of osteoblasts, was assessed qualitatively and quantitatively within rBMSCs on all the samples. As shown in Figure 11A, the ALP-positive area on all the samples after 7 days was much larger than that after 4 days, whereas no significant difference was observed between the SLA and the Ag-PIII groups. The results are corroborated by the quantitative analysis shown in Figure 11B, suggesting that Ag-PIII did not hinder the osteoblastic differentiation of rBMSCs in terms of ALP production.

\section{Discussion}

SLA treatment is commonly used to produce microrough surfaces for dental implants and obtain superior osseointegration properties. ${ }^{4,5}$ However, constant risks of implant-related infection have become the biggest problem for SLA-treated titanium due to its high susceptibility to bacterial adhesion and biofilm formation. ${ }^{10,11} \mathrm{Ag}$ NPs have promising antibacterial activity but the small size and high mobility raise safety concerns due to their potential cytotoxicity. ${ }^{26}$ In this study, size tunable Ag NPs were fabricated and incorporated onto SLA surfaces by manipulating the ASH effect of Ag-PIII. It was found that immobilization of Ag NPs on SLA-treated titanium gave rise to both good antibacterial activity and excellent compatibility with mammalian cells (Figure 12).

Various analytical methods demonstrated that the microbiota associated with peri-implant disease was mixed, 
A

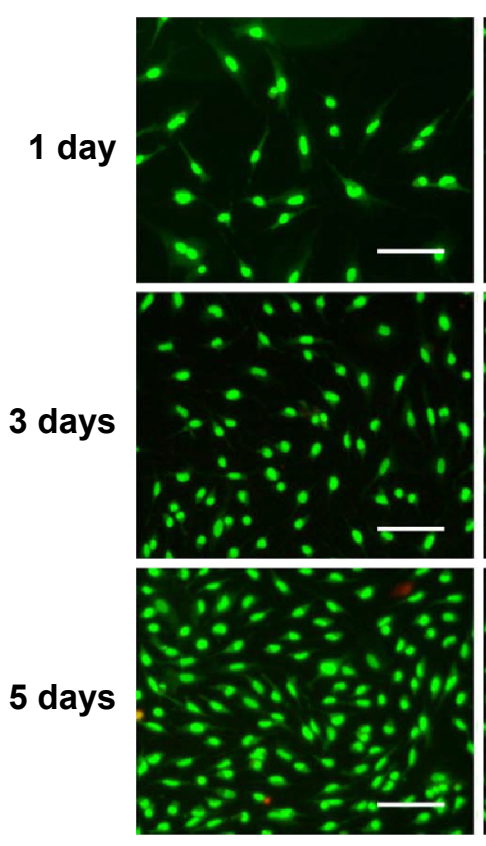

$30 \mathrm{~min}-15 \mathrm{Ag}-\mathrm{PIII}$
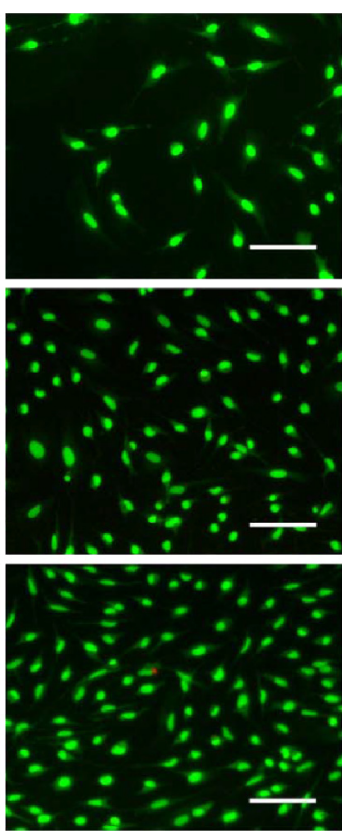

60 min-15 Ag-PIII
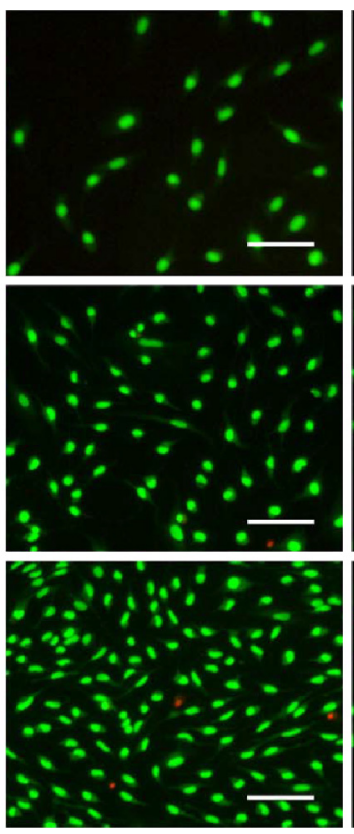

90 min-15 Ag-PIII

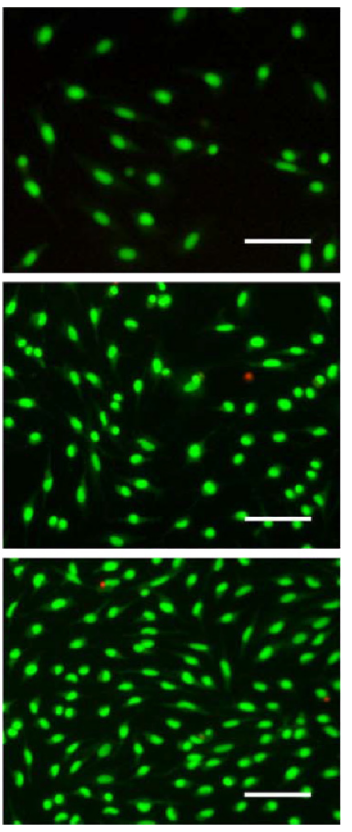

B

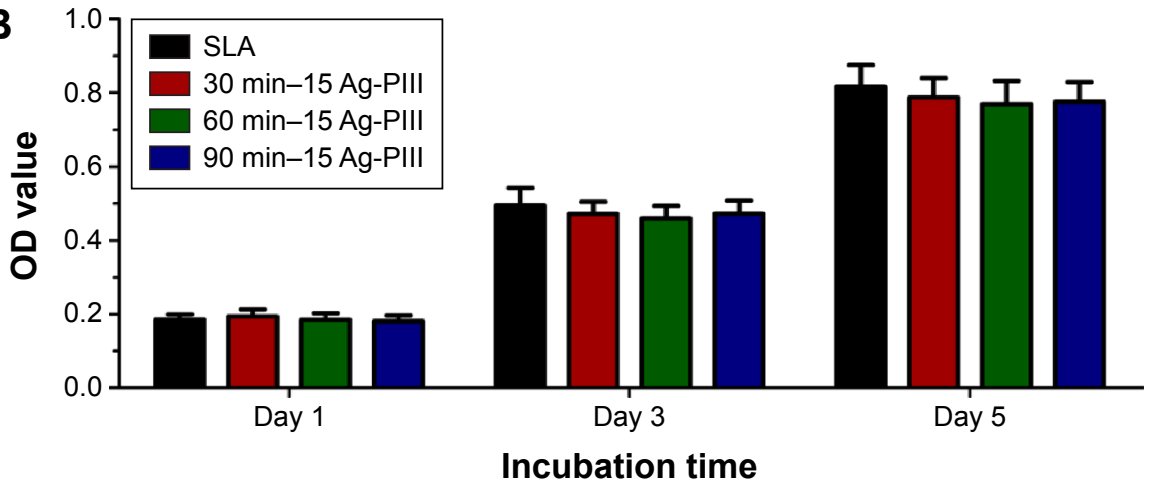

Figure 10 (A) Fluorescence images of Live/Dead staining of rBMSCs after culturing on SLA, 30 min-I5 Ag-PIII, 60 min-I5 Ag-PIII, and 90 min-I5 Ag-PIII for I day, 3 days, and 5 days. (B) Cell proliferation assay of rBMSCs on various groups.

Notes: (A) The scale bar represents $200 \mu \mathrm{m}$. (B) No significant differences in cell proliferation were found between SLA and Ag-PIII groups after culturing for I day, 3 days, and 5 days. All the data are expressed as means \pm standard deviations $(n=3)$. 30 min- 15 Ag-PIII, titanium surfaces treated by first SLA procedure and then silver plasma immersion ion implantation at $15 \mathrm{kV}$ for 30 minutes; 60 min- $15 \mathrm{Ag}$-PIII, titanium surfaces treated by first SLA procedure and then silver plasma immersion ion implantation at $15 \mathrm{kV}$ for 60 minutes; 90 min-15 Ag-PIII, titanium surfaces treated by first SLA procedure and then silver plasma immersion ion implantation at I5 kV for 90 minutes. Abbreviations: rBMSCs, rat bone marrow mesenchymal stem cells; SLA, sand-blasted, large grit, and acid etched; Ag-PIII, silver plasma immersion ion implantation; min, minutes.

somewhat variable and, in most cases, dominated by diverse gram-negative anaerobic bacteria, among which $F$. nucleatum was a typical one. ${ }^{54}$ In addition to the commonly suspected pathogens for peri-implant disease, a number of clinical studies have identified high levels of $S$. aureus within deep peri-implant pockets that were suppurative and display bleeding on probing. ${ }^{48}$ Other studies also evidenced that $S$. aureus exhibited a specific affinity to titanium surfaces. ${ }^{55}$ Therefore, $S$. aureus (gram-positive bacteria) and F. nucleatum (gramnegative bacteria) were utilized for antibacterial evaluations. Furthermore, it was documented that implants were most susceptible to surface bacteria colonization during the initial
6 hours after implantation, ${ }^{56}$ which confirmed that the antimicrobial effects in the first day were critical to implants. In this study, various antimicrobial assays consistently showed that Ag NPs immobilized on SLA-treated titanium by Ag-PIII are effective in inhibiting the proliferation of both pathogenic microbes within the first day. And the F. nucleatum was more susceptible to Ag NPs in comparison to S. aureus. It is well established that antibacterial activities were relevant to the interaction between deterrents and cell wall of bacteria. ${ }^{57,58}$ The cell wall in gram-positive $S$. aureus is constructed by an inner cell membrane and an outer thick layer of peptidoglycan, ${ }^{59}$ whereas that in gram-negative $F$. nucleatum presents an inner 

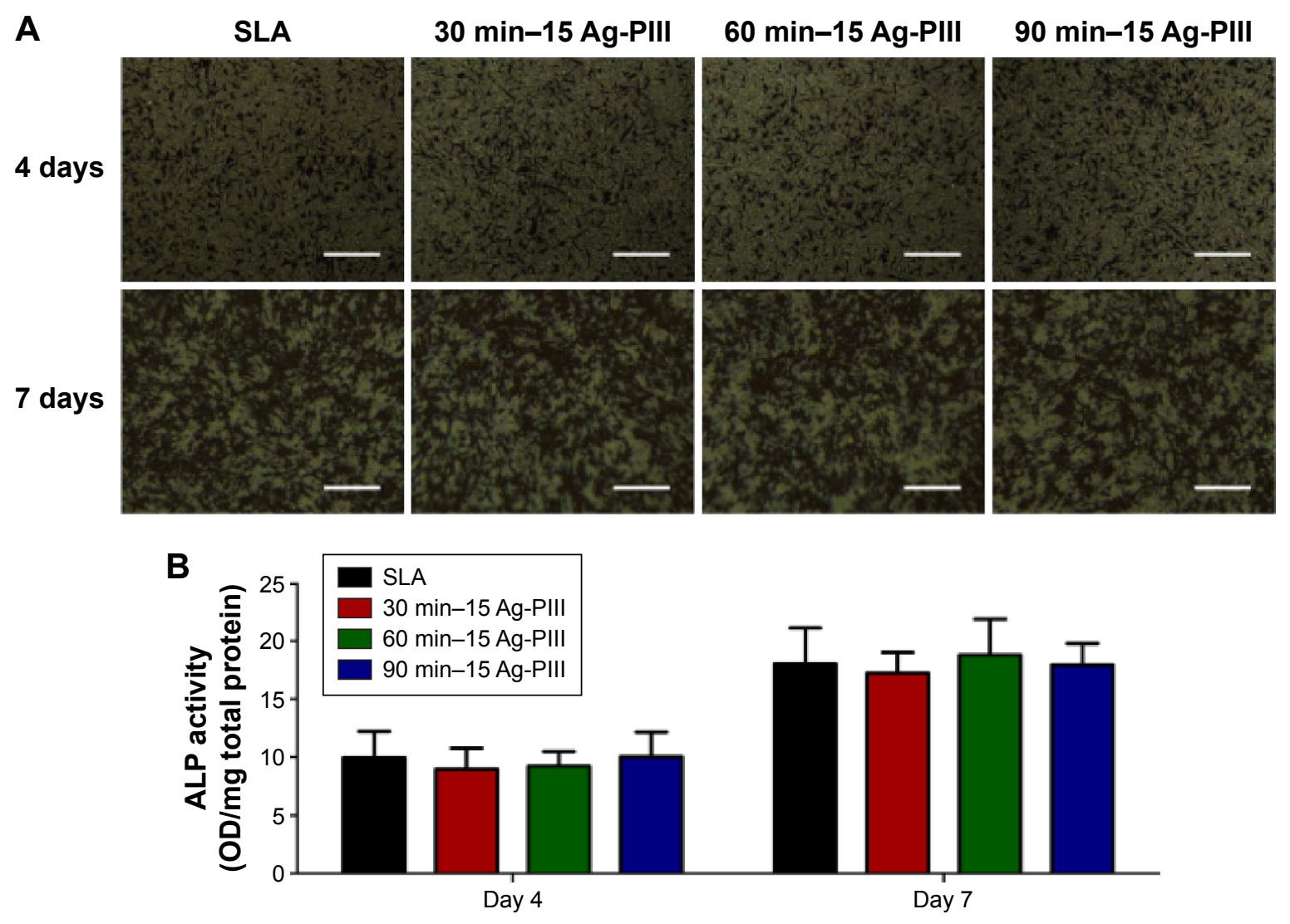

Culture time

Figure II (A) ALP-positive areas of rBMSCs cultured on SLA, 30 min-I5 Ag-PIII, 60 min-15 Ag-PIII, and 90 min-I5 Ag-PIII for 4 days and 7 days and (B) corresponding colorimetrically qualitative results.

Notes: (A) The scale bar represents $300 \mu \mathrm{m}$. (B) No significant differences in ALP production are found between SLA and Ag-PIII groups after culturing for 4 days and 7 days. All the data are expressed as means \pm standard deviations $(n=3)$. 30 min- 15 Ag-PIII, titanium surfaces treated by first SLA procedure and then silver plasma immersion ion implantation at $15 \mathrm{kV}$ for 30 minutes; 60 min-15 Ag-PIII, titanium surfaces treated by first SLA procedure and then silver plasma immersion ion implantation at I5 $\mathrm{kV}$ for 60 minutes; 90 min- 15 Ag-PIII, titanium surfaces treated by first SLA procedure and then silver plasma immersion ion implantation at $15 \mathrm{kV}$ for 90 minutes.

Abbreviations: ALP, alkaline phosphatase; rBMSCs, rat bone marrow mesenchymal stem cells; SLA, sand-blasted, large grit, and acid etched; Ag-PIII, silver plasma immersion ion implantation; min, minutes.

membrane covered by just a thin layer of peptidoglycan and an outer layer of lipopolysaccharides. ${ }^{60}$ Accordingly, the higher susceptibility of gram-negative bacteria could be interpreted in terms of their less rigid cell wall structure, which is more active to Ag NPs irrespective of the mechanism of action (either the release of $\mathrm{Ag}^{+}$or direct contact). In addition, S. aureus contains more potent detoxification agents, such as golden carotenoid pigments and catalase, to resist oxidative stress, ${ }^{61}$ which may grant the bacteria more resistance and insusceptibility than $F$. nucleatum under the same damaging action of Ag NPs.

Bacterial contamination may occur not only perioperatively but also hematogenously later during the lifetime of the implant, indicating that longevity and safety are crucial to clinical adoption. ${ }^{51}$ This problem cannot be circumvented neither by a releasing strategy because the antibacterial agent will run out eventually nor by a long-term administration of prophylactic antibiotics due to the risk of resistance development. ${ }^{24}$
Thus, Ag NPs were immobilized onto the SLA surface using the Ag-PIII technique. Here, it was found that the antibacterial activity of the Ag-PIII samples does not deteriorate either after incubation in PBS for up to 30 days or after multiple cycles of bacteria exposure. The stable and long-lasting antibacterial effects rendered by Ag-PIII groups were very likely owing to that the immobilized Ag NPs inhibited bacterial cell via a continuous direct contact manner rather than a release killing path. Moreover, it is worth mentioning that in our antibacterial assay, the samples were subject to intense bacteria attack by immersing in $1 \mathrm{~mL}$ of the bacterial suspension containing $10^{6} \mathrm{CFU} / \mathrm{mL}$ or $10^{7} \mathrm{CFU} / \mathrm{mL}$. These conditions were much harsher than the normal situation in vivo. ${ }^{25}$ Consequently, the samples that showed excellent antibacterial ability during the first 30 days were expected to be good for a much longer period of time under normal conditions.

Though highly effective against bacteria, the mechanism of Ag NPs is still controversial. It is generally accepted that 


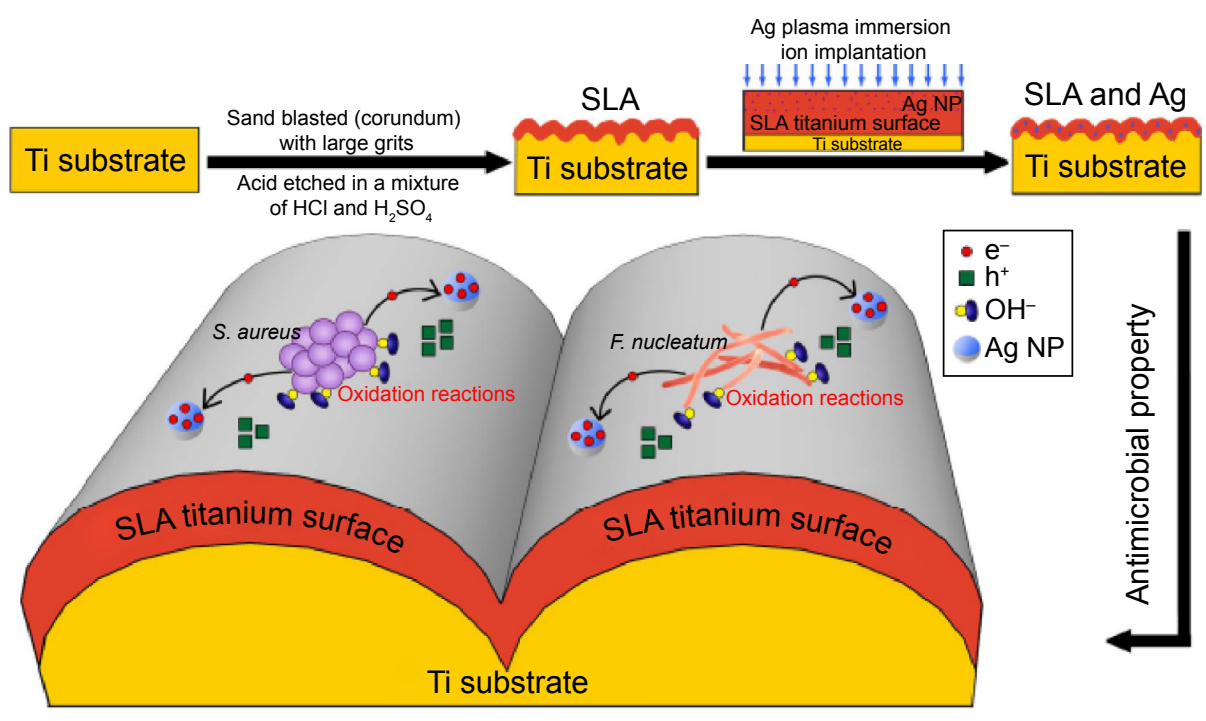

Figure 12 Schematic diagram of the fabrication procedures for the micro/nanostructured titanium and illustration for the possible antibacterial mechanism of the Ag NPs immobilized on SLA-produced titanium oxide layer.

Notes: 30 min-15 Ag-PIII, titanium surfaces treated by first SLA procedure and then silver plasma immersion ion implantation at $15 \mathrm{kV}$ for 30 minutes; 60 min-15 Ag-PIII, titanium surfaces treated by first SLA procedure and then silver plasma immersion ion implantation at $15 \mathrm{kV}$ for 60 minutes; 90 min- 15 Ag-PIII, titanium surfaces treated by first SLA procedure and then silver plasma immersion ion implantation at $15 \mathrm{kV}$ for 90 minutes.

Abbreviations: Ag NPs, silver nanoparticles; SLA, sand-blasted, large grit, and acid etched; Ag-PIII, silver plasma immersion ion implantation; min, minutes.

the antibacterial action of Ag NPs depends on the availability of silver ions $\left(\mathrm{Ag}^{+}\right) .{ }^{62-64}$ Other studies have shown that oxidizing species in lieu of reducing agents might be responsible for the antibacterial effects. ${ }^{65,66}$ In our study, the extremely low silver concentrations in the solution ruled out the inherent toxicity of silver ions $\left(\mathrm{Ag}^{+}\right)$to bacteria and so the inhibition to bacteria was mainly due to the neutral metallic silver $\left(\mathrm{Ag}^{0}\right)$ rendered by Ag NPs. The underlying factors contributing to the antimicrobial results of Ag NPs may be similar to those observed by Cao et al. ${ }^{28}$ More specifically, when Ag NPs (incorporated by Ag-PIII) contact with a semiconductor, such as titanium oxide (produced in the SLA process), a so-called Schottky barrier will be established due to the Fermi level alignment at the metal (Ag NPs)/semiconductor (titanium oxide) interface, endowing metallic silver particles $\left(\mathrm{Ag}^{0}\right)$ with electron trapping capability. Moreover, extracellular electron transport is a common behavior of microbes by which they synthesize adenosine triphosphate for cell growth and maintenance. ${ }^{67}$ The electrons extruded by adherent bacteria can be captured by the immobilized Ag NPs, arousing accumulation of valence-band holes $\left(h^{+}\right)$on the titanium oxide side, and notable oxidation reactions, which disrupt the integrity of bacterial membranes. In our case, the antibacterial activity of 90 min-15 Ag-PIII was superior to that of 30 min-15 Ag-PIII and 60 min-15 Ag-PIII, which might be ascribed to that the electron storage behavior of Ag NPs was size dependent, and larger particles were better at reserving electrons than smaller ones. ${ }^{28}$ However, given its potential cytotoxicity, any antimicrobial properties may remain insignificant if Ag-PIII-treated SLA surfaces fail to support osteogenesis. Previous studies have shown that diffusive Ag NPs could be readily taken up by mammalian cells through endocytosis and macropinocytosis and release of $\mathrm{Ag}^{+}$directly damages intracellular functions. ${ }^{18}$ In this study, the amount of released $\mathrm{Ag}^{+}$is negligible, and the Ag NPs immobilized using Ag-PIII technique showed almost no side effects to rBMSCs in terms of initial adhesion, cell proliferation, and osteoblastic differentiation. It appeared that $\mathrm{Ag}^{+}$is safe and cytocompatible at low concentrations, although high concentrations of $\mathrm{Ag}^{+}$ might induce cytotoxicity. In addition, it is well established that surface topographical characteristic of dental implants was an important influencing factor for cell functions. ${ }^{37,42}$ Bone tissues are hierarchical structures, which are composed of both micro- and nanoscale building blocks. From the biomimetic viewpoint, hierarchical micro/nanostructures, such as Ag-PIII-treated SLA surfaces, may provide a suitable surface for cell functions during a long time as they can mimic the structure of the natural extracellular matrix. Nevertheless, further studies are needed to verify our inference.

Therefore, it appears that Ag NPs-modified SLA surfaces may be a promising choice for dental implants, since $\mathrm{Ag}$ NPs immobilized by using Ag-PIII techniques possessed good bactericidal ability without triggering cytotoxicity. However, the definite relationship between NP size and antibacterial activities still needs to be investigated. In addition, further animal experiments should be designed to evaluate 
whether the antimicrobial properties are retained in vivo and maintained in the long term.

\section{Conclusion}

In summary, Ag NPs were in situ fabricated and immobilized on SLA-treated titanium by using the Ag-PIII technique. The immobilized Ag NPs did not exhibit apparent cytotoxicity but inhibit the proliferation of both gram-positive $S$. aureus and gram-negative $F$. nucleatum. F. nucleatum showed a higher susceptibility to Ag NPs, which might be ascribed to their less rigid cell wall structure. In addition, the antibacterial efficacy of the immobilized Ag NPs was independent of silver release, and they were efficacious in tackling bacteria even after the materials were incubated in PBS for 30 days or exposed to several cycles of bacteria. These results suggested that by using the proper Ag-PIII conditions, titanium implants with hierarchical micro/nanostructures can be endowed with balanced antibacterial and osteogenic functions, which bodes well for safe and prolonged clinical applications.

\section{Acknowledgment}

This work was supported by the National Basic Research Program of China (973 Program, 2012CB933600), National Natural Science Foundation of China (81470782 and 31370962), Shanghai Rising-Star Program (15QA1404100), Youth Innovation Promotion Association CAS (2015204), and the Open Research Fund of State Key Laboratory of Bioelectronics, Southeast University.

\section{Disclosure}

The authors report no conflicts of interest in this work.

\section{References}

1. Le Guehennec L, Goyenvalle E, Lopez-Heredia MA, Weiss P, Amouriq Y, Layrolle P. Histomorphometric analysis of the osseointegration of four different implant surfaces in the femoral epiphyses of rabbits. Clin Oral Implants Res. 2008;19(11):1103-1110.

2. Schwarz F, Wieland M, Schwartz Z, et al. Potential of chemically modified hydrophilic surface characteristics to support tissue integration of titanium dental implants. J Biomed Mater Res B Appl Biomater. 2009; 88(2):544-557.

3. Zinger O, Anselme K, Denzer A, et al. Time-dependent morphology and adhesion of osteoblastic cells on titanium model surfaces featuring scale-resolved topography. Biomaterials. 2004;25(14):2695-2711.

4. Shalabi MM, Gortemaker A, Van't Hof MA, Jansen JA, Creugers NH. Implant surface roughness and bone healing: a systematic review. J Dent Res. 2006;85(6):496-500.

5. Buser D, Schenk RK, Steinemann S, Fiorellini JP, Fox CH, Stich H. Influence of surface characteristics on bone integration of titanium implants. A histomorphometric study in miniature pigs. J Biomed Mater Res. 1991;25(7):889-902.

6. Wennerberg A, Albrektsson T. Suggested guidelines for the topographic evaluation of implant surfaces. Int J Oral Maxillofac Implants. 2000; 15(3):331-344.
7. Lee JH, Kwon YH, Herr Y, Shin SI, Chung JH. Effect of erbiumdoped: yttrium, aluminium and garnet laser irradiation on the surface microstructure and roughness of sand-blasted, large grit, acid-etched implants. J Periodontal Implant Sci. 2011;41(3):135-142.

8. Wennerberg A, Svanborg LM, Berner S, Andersson M. Spontaneously formed nanostructures on titanium surfaces. Clin Oral Implants Res. 2013;24(2):203-209.

9. Lang NP, Jepsen S, Working G. Implant surfaces and design (Working Group 4). Clin Oral Implants Res. 2009;20(suppl 4):228-231.

10. Cho K, Lee SY, Chang BS, Um HS, Lee JK. The effect of photodynamic therapy on Aggregatibacter actinomycetemcomitans attached to surfacemodified titanium. J Periodontal Implant Sci. 2015;45(2):38-45.

11. Esposito M, Murray-Curtis L, Grusovin MG, Coulthard P, Worthington HV. Interventions for replacing missing teeth: different types of dental implants. Cochrane Database Syst Rev. 2007;4:CD003815.

12. Rottman M, Goldberg J, Hacking SA. Titanium-tethered vancomycin prevents resistance to rifampicin in Staphylococcus aureus in vitro. PLoS One. 2012;7(12):e52883.

13. Darouiche RO. Treatment of infections associated with surgical implants. N Engl J Med. 2004;350(14):1422-1429.

14. Esposito M, Hirsch JM, Lekholm U, Thomsen P. Biological factors contributing to failures of osseointegrated oral implants. Eur J Oral Sci. 1998;106(1):527-551.

15. Gristina AG. Biomaterial-centered infection: microbial adhesion versus tissue integration. Science. 1987;237(4822):1588-1595.

16. Gristina AG, Oga M, Webb LX, Hobgood CD. Adherent bacterial colonization in the pathogenesis of osteomyelitis. Science. 1985;228:990-993.

17. Costerton JW, Stewart PS, Greenberg EP. Bacterial biofilms: a common cause of persistent infections. Science. 1999;284(5418):1318-1322.

18. Flores CY, Minan AG, Grillo CA, Salvarezza RC, Vericat C, Schilardi PL. Citrate-capped silver nanoparticles showing good bactericidal effect against both planktonic and sessile bacteria and a low cytotoxicity to osteoblastic cells. ACS Appl Mater Interfaces. 2013;5(8):3149-3159.

19. Wirth SM, Lowry GV, Tilton RD. Natural organic matter alters biofilm tolerance to silver nanoparticles and dissolved silver. Environ $\mathrm{Sci}$ Technol. 2012;46(22):12687-12696.

20. Agarwal A, Weis TL, Schurr MJ, et al. Surfaces modified with nanometerthick silver-impregnated polymeric films that kill bacteria but support growth of mammalian cells. Biomaterials. 2010;31(4):680-690.

21. Zheng Y, Li J, Liu X, Sun J. Antimicrobial and osteogenic effect of Agimplanted titanium with a nanostructured surface. Int J Nanomedicine. 2012;7:875-884

22. Liao J, Anchun M, Zhu Z, Quan Y. Antibacterial titanium plate deposited by silver nanoparticles exhibits cell compatibility. Int J Nanomedicine. 2010;5:337-342.

23. Wang Z, Sun Y, Wang D, Liu H, Boughton RI. In situ fabrication of silver nanoparticle-filled hydrogen titanate nanotube layer on metallic titanium surface for bacteriostatic and biocompatible implantation. Int J Nanomedicine. 2013;8:2903-2916.

24. Qin $\mathrm{H}, \mathrm{Cao} \mathrm{H}$, Zhao Y, et al. In vitro and in vivo anti-biofilm effects of silver nanoparticles immobilized on titanium. Biomaterials. 2014; 35(33):9114-9125.

25. Zhao L, Wang H, Huo K, et al. Antibacterial nano-structured titania coating incorporated with silver nanoparticles. Biomaterials. 2011;32(24): $5706-5716$.

26. De Giglio E, Cafagna D, Cometa S, et al. An innovative, easily fabricated, silver nanoparticle-based titanium implant coating: development and analytical characterization. Anal Bioanal Chem. 2013;405(2-3):805-816.

27. Navarro E, Piccapietra F, Wagner B, et al. Toxicity of silver nanoparticles to Chlamydomonas reinhardtii. Environ Sci Technol.2008;42(23): 8959-8964.

28. Cao H, Qiao Y, Liu X, et al. Electron storage mediated dark antibacterial action of bound silver nanoparticles: smaller is not always better. Acta Biomater. 2013;9(2):5100-5110.

29. Wu S, Liu X, Yeung A, et al. Plasma-modified biomaterials for self-antimicrobial applications. ACS Appl Mater Interfaces. 2011;3(8): 2851-2860. 
30. Cao H, Liu X, Meng F, Chu PK. Biological actions of silver nanoparticles embedded in titanium controlled by micro-galvanic effects. Biomaterials. 2011;32(3):693-705.

31. Shibata Y, Suzuki D, Omori S, et al. The characteristics of in vitro biological activity of titanium surfaces anodically oxidized in chloride solutions. Biomaterials. 2010;31(33):8546-8555.

32. Biggerstaff JP, Le Puil M, Weidow BL, et al. New methodology for viability testing in environmental samples. Mol Cell Probes. 2006;20(2):141-146.

33. Tan H, Peng Z, Li Q, Xu X, Guo S, Tang T. The use of quaternised chitosan-loaded PMMA to inhibit biofilm formation and downregulate the virulence-associated gene expression of antibiotic-resistant staphylococcus. Biomaterials. 2012;33(2):365-377.

34. Gao A, Hang R, Huang X, et al. The effects of titania nanotubes with embedded silver oxide nanoparticles on bacteria and osteoblasts. Biomaterials. 2014;35(13):4223-4235.

35. Marsich E, Travan A, Donati I, et al. Biological responses of silver-coated thermosets: an in vitro and in vivo study. Acta Biomater. 2013;9(2): 5088-5099.

36. Jiang X, Zhao J, Wang S, et al. Mandibular repair in rats with premineralized silk scaffolds and BMP-2-modified bMSCs. Biomaterials. 2009;30(27):4522-4532.

37. Zhang W, Li Z, Liu Y, et al. Biofunctionalization of a titanium surface with a nano-sawtooth structure regulates the behavior of rat bone marrow mesenchymal stem cells. Int J Nanomedicine. 2012;7:4459-4472.

38. Zhang W, Zhu C, Ye D, et al. Porous silk scaffolds for delivery of growth factors and stem cells to enhance bone regeneration. PLoS One. 2014; 9(7):e102371.

39. Jin G, Qin H, Cao H, et al. Synergistic effects of dual Zn/Ag ion implantation in osteogenic activity and antibacterial ability of titanium. Biomaterials. 2014;35(27):7699-7713.

40. He LG, Li XL, Zeng XZ, et al. Sinomenine induces apoptosis in RAW 264.7 cell-derived osteoclasts in vitro via caspase-3 activation. Acta Pharmacol Sin. 2014;35(2):203-210.

41. Wang G, Li J, Zhang W, et al. Magnesium ion implantation on a micro/ nanostructured titanium surface promotes its bioactivity and osteogenic differentiation function. Int J Nanomedicine. 2014;9:2387-2398.

42. Zhang W, Zhang X, Wang S, et al. Comparison of the use of adipose tissue-derived and bone marrow-derived stem cells for rapid bone regeneration. J Dent Res. 2013;92(12):1136-1141.

43. Gu YX, Du J, Si MS, Mo JJ, Qiao SC, Lai HC. The roles of PI3K/Akt signaling pathway in regulating MC3T3-E1 preosteoblast proliferation and differentiation on SLA and SLActive titanium surfaces. $J$ Biomed Mater Res A. 2013;101(3):748-754.

44. Gu YX, Du J, Zhao JM, Si MS, Mo JJ, Lai HC. Characterization and preosteoblastic behavior of hydroxyapatite-deposited nanotube surface of titanium prepared by anodization coupled with alternative immersion method. J Biomed Mater Res B Appl Biomater. 2012;100(8):2122-2130.

45. Stepanov AL, Krenn JR, Ditlbacher H, et al. Quantitative analysis of surface plasmon interaction with silver nanoparticles. Opt Lett. 2005; 30(12):1524-1526.

46. Li J, Qiao Y, Ding Z, Liu X. Microstructure and properties of Ag/N dual ions implanted titanium. Surf Coat Tech. 2011;205:5430e6.

47. Roy M, Fielding GA, Beyenal H, Bandyopadhyay A, Bose S. Mechanical, in vitro antimicrobial, and biological properties of plasma-sprayed silver-doped hydroxyapatite coating. ACS Appl Mater Interfaces. 2012; 4(3):1341-1349.
48. Zhuang LF, Watt RM, Mattheos N, Si MS, Lai HC, Lang NP. Periodontal and peri-implant microbiota in patients with healthy and inflamed periodontal and peri-implant tissues. Clin Oral Implants Res. Epub 2014.

49. Park J, Kim J, Singha K, Han DK, Park H, Kim WJ. Nitric oxide integrated polyethylenimine-based tri-block copolymer for efficient antibacterial activity. Biomaterials. 2013;34(34):8766-8775.

50. Mei S, Wang H, Wang W, et al. Antibacterial effects and biocompatibility of titanium surfaces with graded silver incorporation in titania nanotubes. Biomaterials. 2014;35(14):4255-4265.

51. Hickok NJ, Shapiro IM. Immobilized antibiotics to prevent orthopaedic implant infections. Adv Drug Deliv Rev. 2012;64(12):1165-1176.

52. Eckhardt S, Brunetto PS, Gagnon J, Priebe M, Giese B, Fromm KM. Nanobio silver: its interactions with peptides and bacteria, and its uses in medicine. Chem Rev. 2013;113(7):4708-4754.

53. Asharani PV, Hande MP, Valiyaveettil S. Anti-proliferative activity of silver nanoparticles. BMC Cell Biol. 2009;10:65.

54. Albertini M, López-Cerero L, O’Sullivan MG, et al. Assessment of periodontal and opportunistic flora in patients with peri-implantitis. Clin Oral Implants Res. 2015;26(8):937-941.

55. Harris LG, Richards RG. Staphylococci and implant surfaces: a review. Injury. 2006;37(suppl 2):S3-S14.

56. Pritchard EM, Valentin T, Panilaitis B, Omenetto F, Kaplan DL. Antibiotic-releasing silk biomaterials for infection prevention and treatment. Adv Funct Mater. 2013;23(7):854-861.

57. Ma S, Zhan S, Jia Y, Zhou Q. Highly efficient antibacterial and $\mathrm{Pb}$ (II) removal effects of Ag-CoFe2O4-GO nanocomposite. ACS Appl Mater Interfaces. 2015;7(19):10576-10586.

58. Martinez JL. Antibiotics and antibiotic resistance genes in natural environments. Science. 2008;321(5887):365-367.

59. Tang J, Chen Q, Xu L, et al. Graphene oxide-silver nanocomposite as a highly effective antibacterial agent with species-specific mechanisms. ACS Appl Mater Interfaces. 2013;5(9):3867-3874.

60. Tian TF, Shi XZ, Cheng L, et al. Graphene-based nanocomposite as an effective, multifunctional, and recyclable antibacterial agent. $A C S$ Appl Mater Interfaces. 2014;6:8542-8548.

61. Liu GY, Essex A, Buchanan JT, et al. Staphylococcus aureus golden pigment impairs neutrophil killing and promotes virulence through its antioxidant activity. J Exp Med. 2005;202(2):209-215.

62. Juan L, Zhimin Z, Anchun M, Lei L, Jingchao Z. Deposition of silver nanoparticles on titanium surface for antibacterial effect. Int J Nanomedicine. 2010;5:261-267.

63. Matsumura Y, Yoshikata K, Kunisaki S, Tsuchido T. Mode of bactericidal action of silver zeolite and its comparison with that of silver nitrate. Appl Environ Microbiol. 2003;69(7):4278-4281.

64. Chen X, Schluesener HJ. Nanosilver: a nanoproduct in medical application. Toxicol Lett. 2008;176(1):1-12.

65. Lok CN, Ho CM, Chen R, et al. Silver nanoparticles: partial oxidation and antibacterial activities. J Biol Inorg Chem. 2007;12(4):527-534.

66. Feng QL, Wu J, Chen GQ, Cui FZ, Kim TN, Kim JO. A mechanistic study of the antibacterial effect of silver ions on Escherichia coli and Staphylococcus aureus. J Biomed Mater Res. 2000;52(4):662-668.

67. Reguera G, McCarthy KD, Mehta T, Nicoll JS, Tuominen MT, Lovley DR. Extracellular electron transfer via microbial nanowires. Nature. 2005;435:1098-1101.
International Journal of Nanomedicine

\section{Publish your work in this journal}

The International Journal of Nanomedicine is an international, peerreviewed journal focusing on the application of nanotechnology in diagnostics, therapeutics, and drug delivery systems throughout the biomedical field. This journal is indexed on PubMed Central, MedLine, CAS, SciSearch ${ }^{\circledR}$, Current Contents ${ }^{\circledR} /$ Clinical Medicine,

\section{Dovepress}

Journal Citation Reports/Science Edition, EMBase, Scopus and the Elsevier Bibliographic databases. The manuscript management system is completely online and includes a very quick and fair peer-review system, which is all easy to use. Visit http://www.dovepress.com/ testimonials.php to read real quotes from published authors. 